\title{
Synthesis and Characterization of Monodisperse Colloidal Organo-silica Spheres
}

\author{
A. VAN BLAADEREN ${ }^{1}$ AND A. VRIJ \\ Van't Hoff Laboratory, University of Utrecht, Padualaan 8, 3584 CH Utrecht, The Netherlands
}

Received March 9, 1992; accepted August 11, 1992

\begin{abstract}
Monodisperse, colloidal silica spheres were prepared from tetraethoxysilane (TES) in mixtures of ammonia, water, and ethanol. The surface of the particles could be coated through a subsequent chemical reaction with the silane coupling agent 3aminopropyltriethoxysilane (APS). Also, a new kind of monodisperse, colloidal silica spheres (organo-silica spheres) was prepared starting from mixtures of APS and TES. Organo-silica spheres synthesized with equal amounts of APS and TES were found to contain as much as $37 \%$ of the total amount of APS. The amount taken up by the particles, and therefore also the particle properties, depended on the initial composition of the mixture of alkoxides. It is argued that the most important reaction by which APS is incorporated into the particles is through an alcohol-producing condensation reaction with a silanol group bonded to a silicon atom that was part of a TES molecule. Because of this proposed mechanism, it is argued that this basecatalyzed method of making hybrid organic-silica particles will also be applicable to mixtures of other organoalkoxides. The organo-silica spheres differed from silica spheres prepared with TES alone in the following aspects: The negative surface charge in the mixture of water, ammonia and ethanol was reduced, the mass density was lower (e.g., $1.51 \mathrm{~g} / \mathrm{cm}^{3}$ compared to 1.98 $\mathrm{g} / \mathrm{cm}^{3}$ ), the microporosity was larger, and the siloxane structure was less condensed. The particle refractive index was higher, but the differences were small (around 0.02). It was shown that particles with APS on the surface could be grown larger with a silica layer prepared from TES. Organo-silica particles and silicacoated organo-silica particles were surface coated with stearyl alcohol. The resulting stability in several solvents was assessed. The different colloidal systems were characterized by static and dynamic light scattering, transmission electron microscopy, elemental analysis, nitrogen adsorption measurements, electrophoresis, and qualitative ${ }^{13} \mathrm{C}$ and quantitative ${ }^{19} \mathrm{Si}$ solid-state nuclear magnetic resonance spectroscopy. C 1993 Academic Press, Inc.
\end{abstract}

\section{INTRODUCTION}

Monodisperse, submicrometer silica spheres prepared by hydrolysis and condensation of tetraalkoxysilanes according to a method developed by Stöber et al. (1) have been used

\footnotetext{
${ }^{1}$ To whom correspondence should be addressed.
}

as a model system in many different fields such as ceramics, catalysis, chromatography, and colloids. We are interested in the use of silica spheres as a model system to study the properties of concentrated colloidal dispersions. In such studies it is not only a requirement to use stable, monodisperse, unclustered colloids, but it is also of great importance to be able to adjust the surface properties and chemical composition of the particles.

For instance, the coating of the hydrophilic silica surface with octadecanol renders the surface hydrophobic and makes it possible to disperse the colloids in apolar solvents like cyclohexane, toluene, or chloroform. Because the refractive index of silica prepared according to the method of Stöber et al. $\left(n_{\mathrm{p}} \approx 1.45\right)$ is close to that of most apolar organic solvents, multiple scattering can be avoided and concentrated dispersions can be conveniently studied with light scattering (2). Also, particles with the same surface coating but different compositions of the core can be used in tracer studies (3). Furthermore, the apolar character of the surface and refractive index matching of the core make it possible to describe the interparticle interactions in some solvents with a hard sphere potential (4).

Philipse and Vrij showed (5) that it is also possible to use silane coupling agents to prepare stable, surface-coated silica dispersions. Methacryloxypropyltrimethoxysilane coated silica particles were made that could be dispersed in solvents like tetrahydrofuran and mixtures of ethanol and toluene. These solvents are too polar to disperse octadecanol-coated particles. With the surface coating of methacryloxypropyl, a net charge stabilizes the particles. Other examples of the use of silane coupling agents to coat colloidal silica spheres can be found in (6-8). In this work we describe the coating of the surface of silica spheres with 3-aminopropyltriethoxysilane (APS).

We also present a new procedure to synthesize monodisperse spherical particles from mixtures of organo-alkoxysilanes and alkoxysilanes. The particles consist of "hybrid" chemical structures between that of organic-(polymer) and inorganic-(oxide) colloidal particles. These hybrid, organosilica spheres are prepared from mixtures of APS and tetraethoxysilane (TES) using an experimental procedure 
similar to that of Stöber et al., who only used tetraalkoxysilanes. To illustrate the potentials of this new kind of silica spheres, it is shown that these particles can be grown larger with any desired thickness of "pure" silica (seeded growth) just as has been described for "ordinary" silica spheres (9). Furthermore, these layered particles can subsequently be surface coated with octadecanol to make them dispersable in organic solvents. We also show the physical and chemical characterization of such particles.

The influence on the interparticle potential and particle properties, like density and refractive index, of the incorporation of APS into the silica spheres is described and a possible mechanism of the growth of these organo-silica spheres is discussed. Already this kind of particles has been used by us to chemically attach or incorporate other molecules onto/inside silica spheres $(11,12)$.

The coupling agent APS was not only chosen because of the reactivity of the amino group toward other functional groups, but also because it has been often used in composite materials and therefore, there is a large amount of knowledge available in the literature (13-21).

As has already been demonstrated with the characterization of "Stöber" silica, only ${ }^{13} \mathrm{C}$ and ${ }^{29} \mathrm{Si}$ solid-state nuclear magnetic resonance (NMR) can provide information of composite structures on a molecular scale. The characterization of the chemical microstructure and particle morphology of these new organo-silica spheres described in this paper is a continuation of our analytical work described elsewhere (19). Two experiments on the hydrolysis of TES and $\Lambda \mathrm{PS}$ as followed by ${ }^{13} \mathrm{C}$ NMR are included in the present paper, because they provide essential information about the mechanisms of the synthesis. These measurements are part of a larger study of the kinetics of the synthesis of silica according to the method of Stöber $e t$ al.

The prepared colloidal systems were further characterized by light scattering. Light scattering is very sensitive to small amounts of irregular particular materials, like dust or clusters of otherwise monodisperse spheres. Such irregularities are very difficult to detect with visual observation of the dispersion or other techniques, like transmission electron microscopy. Other particle properties that were determined and that are important for the use of these systems in studies of concentrated dispersions (e.g., with light, neutron, or X-ray scattering) are hydrogen and carbon content, density, refractive index, and surface area and porosity. The influence of APS on the charge stabilization was assessed with electrophoresis and salt additions.

In the next section a short outline is given of the present understanding of the base-catalyzed chemistry of organoalkoxysilanes and some of the peculiar behavior of APS. The outline is necessary to understand most of the results obtained in the synthesis of the coated and organo-silica spheres. Further, some remarks are made concerning the use of several solid-state NMR techniques on the silica systems studied.

\section{THEORETICAL}

\section{A. Base-Catalyzed Reactions of Organo-alkoxysilanes}

(Organo-)alkoxysilanes in a mixture of water, ammonia and the cosolvent ethanol may undergo many different reactions. Schematically all the reactions possible at room temperature can be represented by two pathways (other reactions not represented by Eqs. [1] and [2] are considered not to take place):

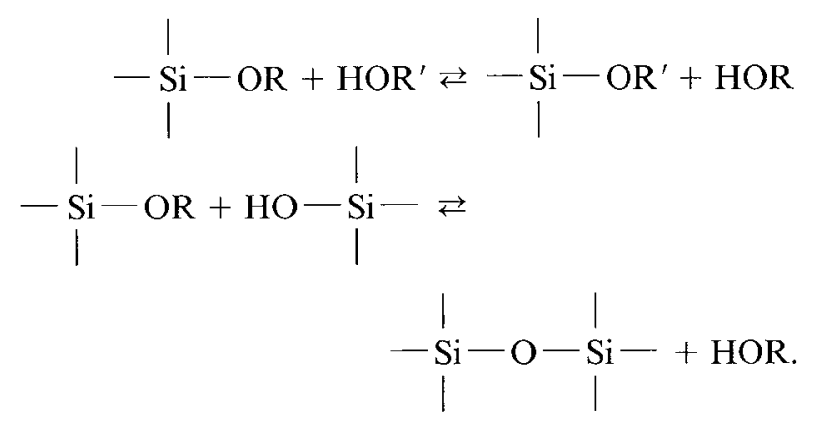

$\mathrm{R}$ and $\mathrm{R}^{\prime}$ stand for a hydrogen atom or an ethyl group. The bonds extending from the silicon atoms to atoms not depicted can be part of an ethoxy group, a silanol group, a 3-aminopropyl group, or a siloxane bond with another silicon atom. In order to distinguish between the silicon atoms bonded with different groups, a special nomenclature has been developed (20). A silicon atom bonded through siloxane bonds with four other silicons is designated the symbol $Q^{4}$ ( $Q$ from quaternary, i.e., having the ability to form four siloxane bonds); if it is bonded with three other silicons atoms $\mathrm{Q}^{3}$, etc. For an organo-alkoxysilane like 3-aminopropyltriethoxysilane, one possibility of forming siloxane bonds is removed through the formation of a $\mathrm{Si}-\mathrm{C}$ bond; such siloxane structures are represented by a $T^{3}, T^{2}$ etc. ( $T$ from ternary or trifunctional).

The reactions covered by Eq. [1] are ester exchange (in this work only the "exchange" between ethoxy and ethanol is possible), hydrolysis, and its reversal, esterification. It is generally accepted, that the base-catalyzed reactions [1] proceed through a bimolecular nucleophilic attack on the silicon atom, resulting in a negatively charged, pentacoordinate transition state $\left(S_{\mathrm{N}} 2\right.$ reaction). Several factors influencing the hydrolysis rate that are of importance to the work described here will be shortly discussed (see Ref. (18) for a complete discussion ).

The nucleophile attacking the silicon atom in the hydrolysis reaction is $\mathrm{OH}^{-}$; an increase in the concentration of this catalyst results in an increase in reaction rate. 'l herefore, the hydrolysis rate increases if the concentration of $\mathrm{NH}_{3}$ and/ or $\mathrm{H}_{2} \mathrm{O}$ is increased. Steric and inductive effects of the substituents on silicon are also important in this respect. Both hydrolysis and condensation decrease the electron density on the silicon atom. In hydrolysis because the more electron- 
withdrawing $\mathrm{OH}$ group replaces $\mathrm{OEth}$. In condensation because OR is substituted for OSi. This decrease in electron density stabilizes the negative charged transition state and leads to an increase in the hydrolysis rate for each subsequent hydrolysis step. Steric hindering effects of the ethoxy groups will increase this trend. Thus, for monomers the hydrolysis of the first alkoxy group is the rate-determining step. Faster hydrolysis is also expected for more condensed species; in this case steric effects, however, work in the opposite direction and are probably more important for larger siloxane moieties.

Replacement of an ethoxy or hydroxy group with an alkyl group results in the opposite inductive effect: the electron density on silicon is increased. The importance of this destabilizing effect on the base catalyzed hydrolysis is illustrated by comparing the reaction rates of TES and methylethoxysilanes in a mixture of water and ammonia in ethanol. $\mathrm{Hy}-$ drolysis of TES is essentially complete in $10 \mathrm{~min}$, while methyltriethoxysilane has been converted to methyltrihydroxysilane for only $10 \%$ during this reaction time (10).

The condensation reactions [2] producing water or ethanol, also take place through a base catalyzed nucleophilic attack on silicon, which results in a pentavalent transition state with negative charge. Because of the resemblance with the hydrolysis mechanism, the influence on the reaction rate of $\left[\mathrm{H}_{2} \mathrm{O}\right],\left[\mathrm{NH}_{3}\right]$, and the inductive effects of the substituents on silicon are very similar (21). However, for an understanding of the formation of condensed colloidal silica, it is important to bear in mind that for basc-catalyzed systems, the siloxane bond formation is much faster than the hydrolysis of the alkoxide.

Also important for the formation of highly condensed siloxane structures is the acidity of the different silanol groups. The nucleophile in the condensation reactions is a deprotonated silanol group. The acidity of a silanol group belonging to a more condensed silicon atom (f.i., $\mathrm{Q}^{3}, \mathrm{Q}^{2}$, or $\mathrm{T}^{2}$ ) increases rapidly with the degree of condensation, because of electron withdrawing effects of siloxane bonds. Therefore, the less deprotonated monomer will react preferentially with higher polymerized species (23). All these factors explain why only highly condensed species and monomers are observed during base-catalyzed reactions of ethoxysilanes (24).

Just as in hydrolysis, the electron donating character of an alkyl group reduces the condensation rate of organoalkoxysilanes relative to a tetraalkoxide by destabilizing the transition state. The decreased acidity of the silanol group also decreases the concentration of nucleophiles. For these reasons, it is argued $(21,22)$ that for the production of hybrid materials form tetraalkoxysilanes and organo-alkoxysilanes it is best to use acid-catalyzed reaction schemes. With acidcatalysis all the induction effects are reversed, making the organo-alkoxide the fastest condensing and hydrolyzing species.

Besides all the points mentioned above, which are generally true for organo-alkoxides, APS has some additional properties that make its condensation behavior exceptional. At basic $\mathrm{pH}$ values in water, APS dissolves readily to a solution of unlimited stability, while other organo-alkoxysilanes rapidly condensate to insoluble precipitates. This behavior is explained by Plueddemann, who has investigated aminoalkylalkoxysilanes extensively (25), by assuming that the hydrolyzed APS forms a six- or five-membered chelate ring that sterically hinders condensation.

\section{B. Solid-State Nuclear Magnetic Resonance Spectroscopy (NMR)}

${ }^{13} \mathrm{C}$ and ${ }^{29} \mathrm{Si}$ solid-state NMR spectroscopy is a powerful method to characterize the chemical structure of substances that can be prepared from (organo)alkoxysilanes (26). In the solid state, the linewidth is increased owing to dipoledipole interactions and the anisotropy of the chemical shift tensor. Magic angle spinning (MAS) and high-power decoupling make it possible to obtain high-resolution spectra. These techniques perform the averaging of the dipolar interactions and chemical shift anisotropy that is achieved in a liquid by the rapid motions of the molecules with respect to the magnetic field. By manipulation of the spins in the rotating frame, the transfer of magnetization from ${ }^{1} \mathrm{H}$ nuclei to ${ }^{13} \mathrm{C}$ or ${ }^{29} \mathrm{Si}$ nuclei becomes possible. This transfer of magnetization, mainly through dipolar interactions, is called cross-polarization (CP), and leads to an increased signal intensity. Contrary to direct excitation of ${ }^{13} \mathrm{C}$ and ${ }^{29} \mathrm{Si}$ nuclei, the necessary delay between acquisitions is now determined by the relatively short spin-lattice relaxation time $\left(T_{1}\right)$ of the protons and not by the long relaxation times of ${ }^{13} \mathrm{C}$ or ${ }^{29} \mathrm{Si}$ nuclei, which is a further advantage of CP.

Lippmaa et al. (27) were among the first to show that the chemical shifts of the ${ }^{29} \mathrm{Si}$ in solid silicates were approximately equal to the chemical shifts of species in solution. Therefore, it is easy to differentiate between $\mathrm{Q}^{4}, \mathrm{Q}^{3}$, and $\mathrm{Q}^{2}$ silicons, because the chemical shifts lie approximately $10 \mathrm{ppm}$ apart $\left(Q^{4}\right.$ at $-110 \mathrm{ppm}, \mathrm{Q}^{3}$ at $-100 \mathrm{ppm}$, etc.). Exchange of one $\mathrm{Si}-\mathrm{O}$ bond against a $\mathrm{Si}-\mathrm{C}$ bond (the transformation of a $\mathrm{Q}$ silicon into a T-silicon ) causes a shift of about $45 \mathrm{ppm}$ and again there is a separation of approximately $10 \mathrm{ppm}$ between the silicons $T^{3}, T^{2}$, and $T^{1}$. Thus, $T^{3}$ can be found close to $-110+45=-65 \mathrm{ppm}$ and $\mathrm{T}^{1}$ at $-65+20=-45 \mathrm{ppm}$ (see Ref. (28) for a literature survey of the chemical shift values of $Q$ and $T$ species).

Maciel and coworkers showed in a series of papers on the characterization of silylated silica gel with ${ }^{13} \mathrm{C}$ and ${ }^{29} \mathrm{Si} \mathrm{CP}-$ MAS NMR, that it is possible to obtain quantitative information about the amount of coverage and the type and number of the bonds that have been formed in the coating reactions (29). To obtain these quantitative results, however, it was necessary to determine in a quantitative way all the parameters that influence the transfer of magnetization in a $\mathrm{CP}$ experiment. 
As was stated above, CP is a selective method to excite nuclei. $\mathrm{Q}^{4}$ silicons far away from protons can never be detected with CP. It was, therefore, argued by Fyfe et al., not to use $\mathrm{CP}$ excitation but direct pulse excitation of ${ }^{29} \mathrm{Si}$ nuclei to obtain quantitative results on all the silicon nuclei (30). To use direct excitation, the long spin-lattice times of ${ }^{29} \mathrm{Si}$ have to be measured first.

In a previous paper ( $19 \mathrm{~b}$ ) we showed, that in silica prepared according to the method of Stöber et al. a selective CP detection of ${ }^{29} \mathrm{Si}$ nuclei close to the particle surface is not possible. These kind of silica spheres consist of a not fully condensed siloxane structure, typically $65 \%$ is $\mathrm{Q}^{4}$, and contain even ethoxy groups inside the particle. Thus, almost all silica nuclei are close to protons. However, it was also shown, that there is a distribution in the values of the parameters characterizing the $\mathrm{CP}$ in one silica system. Furthermore, different silica's were characterized by different parameters. Therefore, for quantitative measurements with $\mathrm{CP}$ all the $\mathrm{CP}$ parameters would have to be determined for each sample. These observations about CP efficiency differences among different samples were recently also reported for native and monofuncially derivatized silica gels (31).

Because of the findings mentioned above, we chose for quantitative ${ }^{29} \mathrm{Si}$ experiments by direct pulse excitation, despite the long ${ }^{29} \mathrm{Si}$ spin-lattice times.

${ }^{13} \mathrm{C}$ CP-MAS NMR measurements were used to qualitatively characterize the fate of the ethoxy groups of the alkoxysilanes and the presence of the aminopropyl groups from the coupling agent.

\section{EXPERIMENTAL}

The following rationale was used to name the colloidal systems. The silica core is referred to as A $x$ with $x$ being the same numeral as was used in (19) to label the same batch of silica particles. An organo-silica core, made from a mixture of APS and TES, is referred to as Mix $x$ where the numeral $x$ is again used to distinguish between different batches. For organo-silica spheres that were grown larger by addition of TES, A $x$ was added to the name, where $x$ referred to the number of additions of TES. The coatings were designated by addition of another letter: $\mathrm{S}$ for a stearyl coating and APS for a coating with the coupling agent. Thus, Mix 3 A3S stands for a particle with an organo-silica core, batch 3 , a silica layer deposited by three additions of TES and an octadecanol coating.

\section{A. Materials}

Ethanol (Merck), $n$-propanol (Baker), 2-bromoethanol (Janssen), toluene (Baker), chloroform (Baker), $\mathrm{LiNO}_{3}$ (Merck), HCL (Merck, 37\%), $\mathrm{Na}_{2} \mathrm{SO}_{4}(\mathrm{BDH}), \mathrm{NaCl}$ (Merck, ultrapure), and $\mathrm{AgNO}_{3}$ (Baker) were of analytical reagent quality. Only for the large scale (9:1) synthesis of
A1 absolute technical grade ethanol (Nedalco) was used. Ethanol, 3-aminopropyltriethoxysilane (APS, Janssen) and tetraethoxysilane (TES, Fluka, purum grade) were freshly distilled before each synthesis. Ammonium hydroxide (Merck, 25\%, subsequently referred to as "ammonia") was of analytical reagent quality and contained $14.0 \mathrm{~mol} /$ liter $\mathrm{NH}_{3}$ as indicated by titration.

\section{B. Particle Synthesis}

The silica particles used as the core in the coating reactions with APS were synthesized according to the method of Stöber et al. (1), this synthesis was described in a previous paper (19). The concentrations used are given in lable 1. All the concentrations in Table 1 were calculated assuming all the added liquid volumes were additive. Weights of substances were converted to volumes by density data at $20^{\circ} \mathrm{C}$ from (32). Mixtures of ethanol and water show some volume contraction upon mixing; the calculated concentrations may, therefore, only be used for comparisons with other data in $\mathrm{mol} / \mathrm{dm}^{3}$ given in the literature, and which were calculated using the same procedure.

The coating of A1 and A5 with APS to A1APS and A5APS was done using a similar procedure as described by Philipse et al. (5), who used the coupling agent 3-methacryloxypropyltrimethoxysilane to coat colloidal silica particles. After addition of APS to $400 \mathrm{ml}$ of the reaction mixture in which the silica particles were synthesized (Table 1), the solution was stirred slowly during an hour. After two hours of refluxing, the reaction mixture was concentrated to $150 \mathrm{ml}$ by slow distillation of $250 \mathrm{ml}$ solvent during an hour. Unreacted

TABLE 1

Reactant Concentrations

\begin{tabular}{llccc}
\hline System & $\begin{array}{c}{[\text { [IES] }} \\
(M)\end{array}$ & $\begin{array}{c}\text { [APS] } \\
(M)\end{array}$ & $\begin{array}{c}{\left[\mathrm{H}_{2} \mathrm{O}\right]} \\
(M)\end{array}$ & $\begin{array}{c}{\left[\mathrm{NH}_{3}\right]} \\
(M)\end{array}$ \\
\hline A1 & 0.155 & & 0.855 & 0.318 \\
A1APS & & 0.477 & React. mixt. A1 \\
A4 & 0.167 & & 1.58 & 0.512 \\
A5 & 0.159 & & 3.01 & 1.12 \\
A5APS & & 0.205 & React. mixt. A5 \\
A6 & 0.160 & & 2.65 & 0.986 \\
A6APS & & & React. mixt. A6 \\
Mix1 & 0.0809 & 0.0774 & 2.34 & 0.870 \\
Mix2 & 0.121 & 0.0387 & 2.34 & 0.870 \\
Mix3 & 0.0795 & 0.0760 & 2.96 & 1.10 \\
Mix3A1 & 0.0023 & & 2.96 & 1.10 \\
Mix3A2 & 0.0134 & & 2.96 & 1.10 \\
Mix3A3 & 0.145 & & 2.96 & 1.10 \\
Mix4 & 0.111 & 0.0423 & 3.75 & 1.40 \\
Mix5 & 0.0970 & 0.0557 & 3.75 & 1.40 \\
Mix6 & 0.0912 & 0.0613 & 3.75 & 1.40 \\
Mix7 & 0.0809 & 0.0774 & 2.34 & 0.870 \\
\hline
\end{tabular}

${ }^{a}$ Reaction temperature $25^{\circ} \mathrm{C}$, all other reactions at $20^{\circ} \mathrm{C}$. 
APS was removed by three centrifugation (Beckmann type L5-50B; A1 APS at about $10,000 \mathrm{~g}$, A5APS at about $200 \mathrm{~g}$ ) and redispersing steps in absolute ethanol.

The organo-silica spheres Mix1, Mix2, Mix4, Mix5, and Mix6 were made by adding under vigorous stirring first APS and then TES to the thermostatted $\left(20^{\circ} \mathrm{C}\right)$ mixture of ammonia and water in ethanol. After approximately $12 \mathrm{~h}$ of slow stirring, the particles were transferred to absolute ethanol by centrifugation (around $200 \mathrm{~g}$ ) and redispersing steps, thereby removing unreacted APS. Colloidal crystals were seen in the upper sediment layer of Mix4, Mix5, and Mix6. The crystal layer had a thickness of a few mm.

Mix7 was made just as Mix 1, only now the APS was allowed to hydrolyze before the TES was added. This was done by slowly stirring the reaction mixture containing APS (Table 1) for $24 \mathrm{~h}$. After one day the solution was still completely clear and no scattering of laser light was seen, which indicated that no large structures were present. Already a few minutes after the addition of TES, gelly flocculates appeared. These gellike structures were clearly visible with the eye, but did not scatter much light.

In the synthesis of Mix 3 only one centrifugation step was used to remove the APS and the particles were not redispersed in ethanol, but in the same volume of the solvent mixture that was used in the synthesis (Table 1).

The coating of these organo-silica spheres with silica by TES was started by adding only a very small quantity of TES to prevent the flocculation of the organo-silica spheres (see the results on particle stability). The resulting particles are referred to as Mix $3 \mathrm{~A} 1$. If the total amount of TES (necessary to prepare Mix 3A3) would have been added at once-as can be done without problems when ordinary silica particles are grown larger (9) - the colloidal system would have flocculated in minutes. This was observed in preliminary experiments.

The resulting Mix3Al particles were grown larger by the addition of another somewhat larger quantity of TES to obtain Mix3A2. Again a stable dispersion was obtained after $12 \mathrm{~h}$. The negative result of the test reaction with salicylaldehyde (see below under the section on the determination of APS) indicated that all the amine groups were now buried inside the particle, therefore, a large amount of TES was added (Mix3A3). No signs of flocculation were observed in the suspensions.

A part of the batches of Mix3, Mix3A2, and Mix3A3 particles was subsequently coated with octadecanol (stearylalcohol) as described in detail by Van Helden et al. (2a), to obtain the systems Mix3S, Mix3A2S, and Mix3A3S. The amount of octadecanol used in the coating reactions in grams was 1.25 times the amount of the alkoxides (both TES and APS) in $m l$. A concentrated solution containing $1 \mathrm{~g}$ octadecanol per $2 \mathrm{ml}$ ethanol was prepared by dissolving the octadccanol at $60^{\circ} \mathrm{C}$. In small portions the reaction mixture containing the silica particles was added, and the water, am- monia, and ethanol were distilled away, leaving a stable dispersion of silica in a melt of octadecanol. Under a slow stream of dry nitrogen the temperature of the melt was brought to $190^{\circ} \mathrm{C}$. The esterification of the surface was continued for three hours under slow stirring. Once coated with octadecanol, the silica particles form a gel phase in the octadecanol melt at temperatures around $80^{\circ} \mathrm{C}$. By allowing this gel phase to settle overnight at $80^{\circ} \mathrm{C}$ and decanting the clear octadecanol, most of the unreacted octadecanol could be removed. The rest of the octadecanol was removed by washings in cyclohexane and redispersing the particles after centrifugation at about $200 \mathrm{~g}$ in four steps total. A fraction of the particles was also transferred to toluene and chloroform by this method.

\section{Particle Size and Morphology}

Light scattering. Light scattering measurements were made at $25.0 \pm 0.1{ }^{\circ} \mathrm{C}$ on very dilute, dust free dispersions in ethanol. Static light scattering (SLS) was performed with a Fica-50 photometer using vertically polarized incident and detected light ( $\lambda=436$ and $546 \mathrm{~nm}$ ). A correction was made for scattering of the solvent. Mean intensities as a function of the scattering angle were obtained in the range of $20^{\circ} \leqslant$ $\theta \leqslant 150^{\circ}$. An optical radius (Table 2) was obtained from an analysis of the intraparticle interference in the RayleighGans-Debye approximation as described in (2a, 33).

\section{TABLE 2}

Radii According to Transmission Electron Microscopy and Static and Dynamic I ight Scattering (Stearyl-Coated Particles Dispersed in Cyclohexane, Others in Ethanol)

\begin{tabular}{|c|c|c|c|}
\hline System & $\begin{array}{c}\text { TEM (rel. st. dev.) } \\
(\mathrm{nm})\end{array}$ & SLS (nm) & DLS (nm) \\
\hline Al & $9.01(20 \%)$ & & $9 \pm 2$ \\
\hline AlAPS & $9.15(20 \%)$ & & $14 \pm 3$ \\
\hline A4 & $31.2(13 \%)$ & $40.2 \pm 0.5$ & $36.6 \pm 1.6$ \\
\hline A5 & $62.4(11 \%)$ & $71.5 \pm 0.4$ & $72.1 \pm 1.2$ \\
\hline A5APS & $66.1(11 \%)$ & $73.6 \pm 0.5$ & $80 \pm 3$ \\
\hline A6 & $89.4 \quad(6 \%)$ & $107.2 \pm 0.3$ & $108.1 \pm 0.7$ \\
\hline A6APS & $88.3(6 \%)$ & $109.3 \pm 0.3$ & \\
\hline $\operatorname{Mix} 1$ & $92.2(11 \%)$ & $128 \pm 2$ & $118 \pm 3$ \\
\hline Mix 2 & $78.0 \quad(8 \%)$ & & \\
\hline Mix 3 & $54.6 \quad(11 \%)$ & $62.4 \perp 0.5$ & \\
\hline Mix3S & $53.8 \quad(10 \%)$ & & \\
\hline $\operatorname{Mix} 3 \mathrm{~A} 2$ & & $62.1 \pm 0.5$ & $65 \pm 3$ \\
\hline Mix3A2S & $55.3(10 \%)$ & & \\
\hline $\operatorname{Mix} 3 \mathrm{~A} 3$ & & $80 \pm 1$ & $78 \pm 2$ \\
\hline Mix3A3S & $70.1 \quad(5 \%)^{\circ}$ & & $83 \pm 2$ \\
\hline Mix4 & $165.5 \quad(4 \%)$ & $195 \pm 3$ & $200 \pm 5$ \\
\hline Mix 5 & $123.3(5 \%)$ & $145 \pm 3$ & $150 \pm 4$ \\
\hline Mix6 & $110.3 \quad(5 \%)$ & $135 \pm 3$ & \\
\hline
\end{tabular}

"The few percent "dumbells" (two particles grown together) were nor included in the determination of the polydispersity. 
TABLE 3

Specific Surface Area and Porosity

\begin{tabular}{|c|c|c|c|c|c|c|c|c|}
\hline System & $\begin{array}{c}S_{\mathrm{G}} \\
\left(\mathrm{m}^{2} \mathrm{~g}^{-1}\right)\end{array}$ & $\begin{array}{c}S_{\mathrm{BET}} \\
\left(\mathrm{m}^{2} \mathrm{~g}^{-1}\right)\end{array}$ & $C_{\mathrm{BET}}$ & $\begin{array}{c}S_{\mathrm{T}} \\
\left(\mathrm{m}^{2} \mathrm{~g}^{-1}\right)\end{array}$ & $\begin{array}{c}S_{\mathrm{cum}}{ }^{a} \\
\left(\mathrm{~m}^{2} \mathrm{~g}^{-1}\right)\end{array}$ & $\begin{array}{c}V_{p} \\
\left(\mathrm{~cm}^{3} \mathrm{~g}^{-1}\right)\end{array}$ & $\begin{array}{c}V_{\text {cum }} \\
\left(\mathrm{cm}^{3} \mathrm{~g}^{-1}\right)\end{array}$ & $\begin{array}{c}V_{\mathrm{m}}^{\prime} \\
\left(\mathrm{cm}^{3} \mathrm{~g}^{-1}\right)\end{array}$ \\
\hline A6 & 14 & 22 & 196 & 24 & 13 & 0.093 & 0.089 & 0.010 \\
\hline Mix 3 & 31 & 33 & 41 & 39 & 22 & 0.150 & 0.154 & 0.018 \\
\hline $\operatorname{Mix} 3 A 2$ & 29 & 28 & 162 & 29 & 18 & 0.146 & 0.160 & 0.015 \\
\hline
\end{tabular}

${ }^{a} S_{\text {cum }}$ values were not corrected to take the microporosity into account.

Dynamic light scattering (DLS) results were obtained using an argon ion laser (Spectra Physics Series 2000) operating at 488.0 and $514.5 \mathrm{~nm}$. Auto-correlation functions were measured with a Malvern Multibit K7025 128 points correlator. Diffusion coefficients were obtained from a second order cumulant fit $(34,35)$ using auto-correlation functions obtained from six scattering angles between $35^{\circ}$ and $145^{\circ}$. Except for dispersion A1, the normalized second cumulant yielded values smaller than 0.05 ; the value for $\mathrm{A} 1$ was around 0.12 . A hydrodynamic radius (Table 2) was calculated using the Stokes-Einstein relation. More information about SLS and DLS can be found in Refs. $(36,37)$.

Electron microscopy. Transmission electron micrographs were made by dipping copper 400 -mesh carrier grids in a dilute dispersion. The grids were covered with carbon coated Formvar films and the photographs were made of particles retained on the film. Philips EM301 and Philips CM10 transmission electron microscopes were used, with the magnification calibrated with a diffraction grating.

Particle radii of 300-2000 particles were measured using an interactive image analysis system (IBAS). Assuming a spherical shape, the surface of the particles was used to determine a number averaged particle radius. The relative standard deviation in the particle radius, $\sigma$, was also obtained.

Nitrogen adsorption. The nitrogen adsorption-desorption isotherms were determined at liquid nitrogen boiling temperature $\left(-195^{\circ} \mathrm{C}\right)$ with a Sorptomatic Carlo Erba Serie 1800 apparatus. Measurements were performed on $\approx 1 \mathrm{~g}$ of silica after drying of the particles for three days under nitrogen at $100^{\circ} \mathrm{C}$. The BET specific surface area $\left(S_{\mathrm{BET}}\right)$ and the $C_{\mathrm{BET}}$ constant were calculated from relative pressures in the range 0.05 to 0.33 . A value of $0.162 \mathrm{~nm}^{2}$ was assumed for the surface area of a nitrogen molecule (38).

I he sample pore volume $\left(V_{p}\right)$ is defined as the volume of the liquid equivalent to the gas quantity adsorbed per sample mass unit at the saturation pressure. The $t$-plots (providing $\left.S_{t}\right)$, the cumulative specific surface area $\left(S_{\text {cum }}\right)$, and the pore distributions (providing $V_{\text {cum }}$, the cumulative specific pore volume, and $V_{\mathrm{m}}$, the microporous volume) were all calculated as described in a paper of Lecloux et al. (39). In this paper of Lecloux the characterization with nitrogen adsorption measurements of silica particles prepared according to the Stöber method is fully discussed. The data are collected in Table 3.

\section{Particle Composition}

Solid-state ${ }^{13} \mathrm{C}$ and ${ }^{29}$ Si NMR spectroscopy. High-resolution solid-state NMR spectra were measured at room temperature on a Bruker AM 500 spectrometer (silicon frequency 99.4 MHz, carbon $125.7 \mathrm{MHz}$ ) equipped with a Bruker solid-state accessory. Spectra were obtained using a broadband probehead with a $7-\mathrm{mm}$ double-air-bearing magic-angle-spinning assembly. Spinning speeds around $4000 \mathrm{~Hz}$ were employed. The $90^{\circ}$ pulse lengths for the nuclei ${ }^{13} \mathrm{C}$, ${ }^{29} \mathrm{Si}$, and ${ }^{1} \mathrm{H}$ were around $5.5 \mu$ s. Cross-polarization $(\mathrm{CP}$ ) contact times lay between 0.5 and $4 \mathrm{~ms}$ for carbon, and 0.5 and $6 \mathrm{~ms}$ for silicon; CYCLOPS phase cycling and spin temperature alternation were used to minimize artifacts. The delay time between pulses in the CP experiments was $4 \mathrm{~s}$. The number of accumulated FID's for CP spectra ranged between 200 and 13,000 , depending very much on the system being investigated.

Before the measurements the samples were dried under nitrogen at $90^{\circ} \mathrm{C}$ for $24 \mathrm{~h}$. Typically, the spinner was filled with $300 \mathrm{mg}$ silica. Adamantane and the trimethylsilyl ester of double four-ring octameric silicate, $\mathrm{Q}_{8} \mathrm{M}_{8}$, were used to optimize experimental parameters and as external secondary chemical shift reference relative to tetramethylsilane for ${ }^{13} \mathrm{C}$ and ${ }^{29} \mathrm{Si}$, respectively. Chemical shift values are given in parts per million.

The ${ }^{29} \mathrm{Si}$ nuclei were excited by $\mathrm{CP}$ using a pulse sequence developed by Torchia (40) to obtain an estimation of the $T_{1}$ values for the different silicon nuclei. A CP contact time of $4 \mathrm{~s}$ was used.

Based on the $T_{1}$ value, a delay time of $300 \mathrm{~s}$ between successive direct ${ }^{29} \mathrm{Si}$ excitations was chosen. Typically, 250 FID's were accumulated.

Deconvolution of the spectra was performed by adjusting the height, width and frequency of Gaussian lineshapes to obtain the best visual fit. This was done manually using the Bruker programs Glinfit or automatically using NMRi, again using Gaussian lines.

Elemental analysis. Elemental analysis was carried out by Llemental Microanalysis Limited (Devon, U.K.). Prior 
to sending, the samples were dried for $24 \mathrm{~h}$ at $100^{\circ} \mathrm{C}$ under nitrogen. Before the measurements were made they were dried again $3 \mathrm{~h}$ under the same conditions (Table 4).

Titration and qualitative determination of APS. Qualitative determination of APS in solution or on the silica particles was performed through reaction of the amine group with salicylaldehyde (SA). The formation of the bright yellow salicylaldimine could be used to monitor the contents of supernatant to the presence of APS and could also be used to indicate its presence on dried silica. In all cases an excess of SA was used. The use of SA in this way was described by Wadell et al. (14).

Quantitative determinations of APS on the silica surface was done using a titration scheme developed by Gimpel and Unger (16): Approximately $0.7 \mathrm{~g}$ of APS coated silica was washed with ethanol to remove the unreacted APS. After three centrifugation steps no APS could be detected with the reaction with SA. To convert the functional groups into - $\mathrm{NH}_{3}^{+} \mathrm{Cl}^{-}$the APS-silica was washed three times with a solution of $20 \mathrm{ml}$ containing $0.5 \mathrm{M} \mathrm{NaCl}$ and $0.2 \mathrm{M} \mathrm{HCl}$. The excess reagent was washed away three times with $20 \mathrm{ml}$ ethanol and finally, $\mathrm{Cl}^{-}$was libcrated by three washes with $20 \mathrm{ml} 0.5 \mathrm{M}$ sodium sulfate solution. All the washing steps wcre performed by centrifugation and redispersing.

The content of $\mathrm{Cl}^{-}$in the combined sodium sulfate washings was determined by potentiometric titration with $0.1 \mathrm{M}$ silver nitrate solution using a silver electrode as indicator electrode and a calomel electrode as reference electrode. Use was also made of an automatic titration system manufactured by Mettler, consisting of an electrode potential amplifier DK 10, a rate and endpoint controller DK 11 and an automatic burette DV 11 . To enable an easy comparison with the data obtained with elemental analysis, the measured values were converted to weight percentages of nitrogen (Table 5).

TABLE 4

Elemental Analysis and Determination of Nitrogen by Titration (Both in Weight Percentages)

\begin{tabular}{|c|c|c|c|c|}
\hline \multirow[b]{2}{*}{ System } & \multicolumn{3}{|c|}{ Elemental analysis } & \multirow{2}{*}{$\begin{array}{c}\text { Titration } \\
\mathrm{N}(\%)\end{array}$} \\
\hline & $\mathrm{C}(\%)$ & $\mathrm{H}(\%)$ & $\mathrm{N}(\%)$ & \\
\hline $\mathrm{Al}^{b}$ & $1.68 \pm 0.01^{a}$ & $0.97 \pm 0.08$ & & \\
\hline Al & $1.11 \pm 0.02$ & $0.99 \pm 0.08$ & & \\
\hline AlAPS & $7.29 \pm 0.01$ & $2.20 \pm 0.03$ & $2.18 \pm 0.02$ & 2.031 \\
\hline$A 5^{b}$ & $5.07 \pm 0.12$ & $1.66 \pm 0.07$ & & \\
\hline A5APS & $6.03 \pm 0.05$ & $2.05 \pm 0.04$ & $0.32 \pm 0.01$ & 0.304 \\
\hline A 6 & $3.95 \pm 0.04$ & $1.55 \pm 0.08$ & & \\
\hline A6APS & & & & 0.220 \\
\hline Mix 1 & $12.95 \pm 0.02$ & $3.97 \pm 0.07$ & $4.49 \pm 0.05$ & \\
\hline
\end{tabular}

a Errors indicate differences in duplicate results.

${ }^{b}$ Samples underwent the coating conditions.
TABLE 5

Particle Density $(\rho)$ and Refractive Index $\left(n_{\mathrm{p}}, \lambda=589 \mathrm{~nm}, 25.0^{\circ} \mathrm{C}\right)$

\begin{tabular}{|c|c|c|c|}
\hline System & $\begin{array}{c}\rho \\
\left(\mathrm{g} / \mathrm{cm}^{3}\right)\end{array}$ & $n_{\mathrm{p}}$ & Solvent mixture \\
\hline A5 & 1.98 & & \\
\hline \multirow[t]{2}{*}{ A6 } & 2.03 & 1.462 & Ethanol/toluene \\
\hline & & 1.451 & 2-Bromoethanol/ethanol \\
\hline \multirow[t]{2}{*}{ Mix 1} & 1.51 & 1.476 & Ethanol/toluene \\
\hline & & 1.463 & 2-Bromoethanol/ethanol \\
\hline Mix2 & 1.75 & & \\
\hline Mix 3 & 1.54 & 1.463 & Ethanol/toluene \\
\hline $\operatorname{Mix} 3 \mathrm{~A} 2$ & 1.62 & & \\
\hline $\operatorname{Mix} 3 \mathrm{~A} 2 \mathrm{~S}$ & & 1.478 & Cyclohexane/toluene \\
\hline Mix 3 A3 & 1.79 & & \\
\hline Mix 3 A3S & & 1.449 & Cyclohexane/toluene \\
\hline
\end{tabular}

\section{E. Particle Properties}

Particle density and refractive index. Particle densities were determined by drying a known volume of a concentrated dispersion in ethanol under dry nitrogen at $100^{\circ} \mathrm{C}$ for $24 \mathrm{~h}$ and weighing the residue (Table 5). The refractive index of the particles was obtained by a determination of the maximum in the transmission at $590 \mathrm{~nm}$ of a dispersion (volume fraction about $15 \%$ ) as a function of the composition of the solvent mixture. The refractive index of the solvent mixture at which the turbidity was minimal was taken as the mean refractive index of the particle. Refractive indices were measured using an Abbe refractometer thermostatted at $25.0^{\circ} \mathrm{C}$ and transmission measurements were performed using a Shimadzu (Spectronic 200 UV) double beam spectrophotometer also thermostatted at $25.0^{\circ} \mathrm{C}$.

Electrophoresis and particle stability. Electrophoretic mobilities were determined using the moving boundary technique with a Burton apparatus with two added $U$ tubes to prevent contamination with clcctrolysis products (41). Mobilities were measured in solutions with a $\left[\mathrm{NH}_{3}\right]=1.1$ $M$ and $\left[\mathrm{H}_{2} \mathrm{O}\right]=3.0 \mathrm{M}$ in ethanol. The volume fraction of the particles was $5 \times 10^{-4}$ and the field strength applied was $200 \mathrm{~V} / \mathrm{m}$.

For systems that were not investigated with light scattering the following criterion was used to assess the stability of the dispersion: A dispersion was called stable if both it could be redispersed to a homogeneous dispersion a week after sedimentation to the bottom of the container and also if no change in the sedimentation velocity could be observed after redispersion of the sediment. This arbitrary definition was chosen because it was observed sometimes that a sol could not be dispersed after sedimentation, while no irregularities were seen during the sedimentation process. Of course this definition makes only sense for larger particles, but it includes almost all the particles presented in Table 6. 
TABLE 6

Stability ${ }^{a}$ of Octadecanol-Coated Particles $\left(20^{\circ} \mathrm{C},+=\right.$ Stable, $-=$ Unstable $)$

\begin{tabular}{lccc}
\hline & \multicolumn{3}{c}{ Solvent } \\
\cline { 2 - 4 } System & $\begin{array}{c}\text { Cyclohexane } \\
(2.0)^{b}\end{array}$ & $\begin{array}{c}\text { Toluene } \\
(2.4)\end{array}$ & $\begin{array}{c}\text { Chloroform } \\
(4.8)\end{array}$ \\
\hline Mix3S & - & - & + \\
Mix3A2S & - & - & + \\
Mix3A3S & + & + & + \\
\hline
\end{tabular}

${ }^{a}$ Stability criterium is explained in the text.

${ }^{b}$ Dielectric constant of the solvent.

For three different systems the stability in water, ammonia, and ethanol mixtures was assessed by determination of the limiting concentration of $\mathrm{LiNO}_{3}$ at which the dispersion stayed stable according to the above given definition (see Table 7).

\section{F. Hydrolysis Followed by ${ }^{13} \mathrm{C} N M R$}

High-resolution ${ }^{13} \mathrm{C}$ NMR measurements of TES and APS in solution were made on a Bruker AM 500 spectrometer. The ${ }^{13} \mathrm{C} 90^{\circ}$ pulse length was $5 \mu$ s and 240 FID's were accumulated per spectrum. To decrease the spin-lattice relaxation times, $10 \mathrm{mg}$ of the relaxation reagent chromium( III) acetylacetonate $\left(\mathrm{Cr}(\mathrm{acac})_{3}\right)$ was added per $\mathrm{ml}$ of solvent; therefore, repetition times of $0.5 \mathrm{~s}$ could be used.

The hydrolysis of TES and APS was followed in mixtures of ammonia, water and propanol by integration of the decreasing peak intensity of the $\mathrm{C}-\mathrm{O}$ carbon of the ethoxy group and by integration of the increasing intensity of the $\mathrm{C}-\mathrm{O}$ carbon of the liberated ethanol.

The concentrations for the hydrolysis of TES were $\left[\mathrm{H}_{2} \mathrm{O}\right]$ $=1.80 \mathrm{M},\left[\mathrm{NH}_{3}\right]=0.668 \mathrm{M},[\mathrm{TES}]=0.167 \mathrm{M}$; and for APS, $\left[\mathrm{H}_{2} \mathrm{O}\right]=1.76 \mathrm{M},\left[\mathrm{NH}_{3}\right]=0.656 \mathrm{M},[\mathrm{APS}]=0.234 \mathrm{M}$.

\section{RESULTS AND DISCUSSION}

\section{A. Particle Radius and Shape}

Particle radii as determined with three different experimental techniques are collected in Table 2. The agreement between the hydrodynamic radius obtained by dynamic light scattering and the optical radius as obtained by static light scattering is reasonable to good. This means that for all the different systems the assumption about the optical homogeneity of the particles is justified; there can be no regions within the particles with a difference in refractive index that is large compared to the difference between the refractive index of the solvent $\left(n_{\mathrm{s}}\right)$ and the mean refractive index of the particle $\left(n_{\mathrm{p}}-n_{\mathrm{s}} \approx 1.45-1.36 \approx 0.1\right.$, Table 6$)$.

Particularly for the system Mix3A3, which consist of an organo-silica core and a shell of silica, it is of significance that the optical radius is almost equal to the hydrodynamic radius. As was corroborated by the particle refractive index measurements, this can only be explained if the refractive index of the core is close, relative to the refractive index of the solvent, to that of the shell (Table 6). For a more quantitative assessment of the optical homogeneity of the particles optical contrast variation experiments would have to be performed as described in $(2 b, 33)$.

Radii obtained by electron microscopy are systematically smaller than the radii obtained by light scattering; as before (19) this is attributed to shrinkage of the particles under the electron beam.

The difference in the radii of the APS-coated and uncoated particles, as can be estimated from the elemental analysis data, is too small for a reliable determination.

The particle shape and surface roughness of the organosilica spheres follow the same trend as particles prepared from TES alone. Particles with smaller final radii are less monodisperse, less spherical, and the surface roughness is also higher (see Figs. 1a and 1b). The larger spheres prepared with higher concentrations of ammonia and water (Mix4, Mix5, and Mix6; see Fig. 1c) are almost perfect spheres with a smooth surface and a low polydispersity (see also (19)).

It is possible to calculate the increase in radius for the system Mix 3 to Mix3A3. Before doing so, it is instructive to look first at the calculation of the growth of a silica sphere that is grown larger by addition of TES. In this case, the amount of TES ( $V_{\mathrm{TES} 2}$ ) needed to increase the radius from $R_{1}$ to $R_{2}$ is given by

$$
V_{\mathrm{TES} 2}=V_{\mathrm{TES} 1}\left\{\left(R_{2} / R_{1}\right)^{3}-1\right\}
$$

if $V_{\text {TESI }}$ is the volume of TES needed to prepare the particles with radius $R_{1}$. In the derivation of this formula it is assumed that all the added TES grows on the existing particles and that the density of the added layer is the same as that of the core on which it grows. It has been shown elsewhere that these assumptions are correct (9). Indirectly, the independence of the siloxane bond structure as a function of the

TABLE 7

Electrophoresis and Particle Stability in Ethanol/Ammonia/ Water $\left(\left[\mathrm{NH}_{3}\right]=1.1 \mathrm{M},\left[\mathrm{H}_{2} \mathrm{O}\right]=3.0 \mathrm{M}\right.$, Particle Volume Fraction $5 \times 10^{-4}$ )

\begin{tabular}{lcc}
\hline System & $\begin{array}{c}\text { Mobility } \\
\left(10^{-9} \mathrm{~m}^{2} /(\mathrm{Vs})\right)\end{array}$ & $\begin{array}{c}\text { Instability concentration } \\
\mathrm{LiNO}_{3}(\mathrm{mM})\end{array}$ \\
\hline A6 & 7.3 & $4.8 \pm 0.3^{a}$ \\
A6APS & 3.1 & $<2.5$ \\
Mix 1 & 1.5 & $<2.5$ \\
\hline
\end{tabular}

${ }^{a}$ The limits in between which the instability concentration lies are given. 
TABLE 6

Stability ${ }^{a}$ of Octadecanol-Coated Particles $\left(20^{\circ} \mathrm{C},+=\right.$ Stable, $-=$ Unstable $)$

\begin{tabular}{lccc}
\hline & \multicolumn{3}{c}{ Solvent } \\
\cline { 2 - 4 } & $\begin{array}{c}\text { Cyclohexane } \\
\text { System }\end{array}$ & $\begin{array}{c}\text { Toluene } \\
(2.0)^{b}\end{array}$ & $\begin{array}{c}\text { Chloroform } \\
(4.8)\end{array}$ \\
\hline Mix3S & - & - & + \\
Mix3A2S & - & - & + \\
Mix3A3S & + & + & + \\
\hline
\end{tabular}

${ }^{u}$ Stability criterium is explained in the text.

${ }^{b}$ Dielectric constant of the solvent.

For three different systems the stability in water, ammonia, and ethanol mixtures was assessed by determination of the limiting concentration of $\mathrm{LiNO}_{3}$ at which the dispersion stayed stable according to the above given definition (see Table 7).

\section{F. Hydrolysis Followed by ${ }^{13} \mathrm{C} N M R$}

High-resolution ${ }^{13} \mathrm{C}$ NMR measurements of TES and APS in solution were made on a Bruker AM 500 spectrometer. The ${ }^{13} \mathrm{C} 90^{\circ}$ pulse length was $5 \mu$ s and 240 FID's were accumulated per spectrum. To decrease the spin-lattice relaxation times, $10 \mathrm{mg}$ of the relaxation reagent chromium( III ) acetylacetonate $\left(\mathrm{Cr}(\mathrm{acac})_{3}\right)$ was added per $\mathrm{ml}$ of solvent; therefore, repetition times of $0.5 \mathrm{~s}$ could be used.

The hydrolysis of TES and APS was followed in mixtures of ammonia, water and propanol by integration of the decreasing peak intensity of the $\mathrm{C}-\mathrm{O}$ carbon of the ethoxy group and by integration of the increasing intensity of the $\mathrm{C}$ - O carbon of the liberated ethanol.

I he concentrations for the hydrolysis of 'I ES were $\left[\mathrm{H}_{2} \mathrm{O}\right]$ $=1.80 \mathrm{M},\left[\mathrm{NH}_{3}\right]=0.668 \mathrm{M},[\mathrm{TES}]=0.167 \mathrm{M}$; and for APS, $\left[\mathrm{H}_{2} \mathrm{O}\right]=1.76 M,\left[\mathrm{NH}_{3}\right]=0.656 M$, [APS $]=0.234 M$.

\section{RESULTS AND DISCUSSION}

\section{A. Particle Radius and Shape}

Particle radii as determined with three different experimental techniques are collected in Table 2 . The agreement between the hydrodynamic radius obtained by dynamic light scattering and the optical radius as obtained by static light scattering is reasonable to good. This means that for all the different systems the assumption about the optical homogeneity of the particles is justified; there can be no regions within the particles with a difference in refractive index that is large compared to the difference between the refractive index of the solvent $\left(n_{\mathrm{s}}\right)$ and the mean refractive index of the particle $\left(n_{\mathrm{p}}-n_{\mathrm{s}} \approx 1.45-1.36 \approx 0.1\right.$, Table 6$)$.

Particularly for the system Mix3A3, which consist of an organo-silica core and a shell of silica, it is of significance that the optical radius is almost equal to the hydrodynamic radius. As was corroborated by the particle refractive index measurements, this can only be explained if the refractive index of the core is close, relative to the refractive index of the solvent, to that of the shell (Table 6). For a more quantitative assessment of the optical homogeneity of the particles optical contrast variation experiments would have to be performed as described in $(2 b, 33)$.

Radii obtained by electron microscopy are systematically smaller than the radii obtained by light scattering; as before (19) this is attributed to shrinkage of the particles under the electron beam.

The difference in the radii of the APS-coated and uncoated particles, as can be estimated from the elemental analysis data, is too small for a reliable determination.

The particle shape and surface roughness of the organosilica spheres follow the same trend as particles prepared from TES alone. Particles with smaller final radii are less monodisperse, less spherical, and the surface roughness is also higher (see Figs. la and $1 \mathrm{~b}$ ). The larger spheres prepared with higher concentrations of ammonia and water (Mix4, Mix5, and Mix6; see Fig. 1c) are almost perfect spheres with a smooth surface and a low polydispersity ( see also (19)).

It is possible to calculate the increase in radius for the system Mix 3 to Mix3A3. Before doing so, it is instructive to look first at the calculation of the growth of a silica sphere that is grown larger by addition of TES. In this case, the amount of TES ( $V_{\text {TES2 }}$ ) needed to increase the radius from $R_{1}$ to $R_{2}$ is given by

$$
V_{\mathrm{TES} 2}=V_{\mathrm{TES} 1}\left\{\left(R_{2} / R_{1}\right)^{3}-1\right\}
$$

if $V_{\mathrm{TES} 1}$ is the volume of TES needed to prepare the particles with radius $R_{1}$. In the derivation of this formula it is assumed that all the added TES grows on the existing particles and that the density of the added layer is the same as that of the core on which it grows. It has been shown elsewhere that these assumptions are correct (9). Indirectly, the independence of the siloxane bond structure as a function of the

TABLE 7

Electrophoresis and Particle Stability in Ethanol/Ammonia/ Water $\left(\left[\mathrm{NH}_{3}\right]=1.1 \mathrm{M},\left[\mathrm{H}_{2} \mathrm{O}\right]=3.0 \mathrm{M}\right.$, Particle Volume Fraction $\left.5 \times 10^{-4}\right)$

\begin{tabular}{lcc}
\hline System & $\begin{array}{c}\text { Mobility } \\
\left(10^{-9} \mathrm{~m}^{2} /(\mathrm{Vs})\right)\end{array}$ & $\begin{array}{c}\text { Instability concentration } \\
\mathrm{LiNO}_{3}(\mathrm{~m} M)\end{array}$ \\
\hline A6 & 7.3 & $4.8 \pm 0.3^{a}$ \\
A6APS & 3.1 & $<2.5$ \\
Mix 1 & 1.5 & $<2.5$ \\
\hline
\end{tabular}

${ }^{a}$ The limits in between which the instability concentration lies are given. 
particle radius (19) is also an indication for a constant density of added layers.

Going from Mix 3 to Mix $3 \mathrm{~A} 3$, the layer that is added is clearly not of the same density as the particle core. There are no reasons to expect this silica layer to have properties that are much different from the properties of silica prepared from TES alone. Thus, a density of $2 \mathrm{~g} / \mathrm{cm}^{3}$ is assumed (Table 6). From this value and the determination of the siloxane bond structure and elemental analysis of A5, it can be calculated that 1 mole of TES is converted to $34.0 \mathrm{~cm}^{3}$ of silica (see the section in which the elemental analysis results are described). The Mix3 particles have not been investigated by ${ }^{29} \mathrm{Si} \mathrm{NMR}$, but giving the similarity in density (Table 6) it is probably a reasonable assumption to use the data of Mix 1 . These data lead to a value for the conversion of 1 mole of TES to $68.7 \mathrm{~cm}^{3}$ of organo-silica with the composition of Mix 1. In this way the number of particles Mix 3 can bc calculatcd if $63 \mathrm{~nm}$ (Table 2) is taken for the particle radius. Since electron microscopy showed no indication of a second nucleation, all the added TES is used to increase the volume of the existing Mix 3 particles. This extra particle volume with the properties of $\mathrm{A} 5$ leads to a particle radius of $79 \mathrm{~nm}$ for the spheres of Mix $3 \mathrm{~A} 3$. This calculated radius is in excellent agreement with the value obtained with light scattering: $79 \mathrm{~nm}$ (Table 2).

Figure 1d shows a micrograph of the structure obtained in the synthesis of Mix 7 where APS was prehydrolyzed before the TES was added to the reaction mixture. The gel-like structure that resulted seems to consist of very small subparticles (radius around $10 \mathrm{~nm}$ ) that have aggregated during the reaction. In the section on the mechanism of the incorporation of APS this result is discussed further.

\section{B. Specific Surface Area and Porosity}

The specific surface area and sample porosity were determined for the organo-silica particle Mix 3 and for the same particle with an additional thin layer of silica: Mix 3A2. A6 was also measured, to be able to compare the results with an "ordinary" silica particle (Table 3 ). The adsorption-desorption isotherm shapes according to the so called BDDT classification (42) were found to be of type IV, characteristic of mesopores $(2 \mathrm{~nm}<$ diameter $<50 \mathrm{~nm}$ ). However, the shape was also very close to that of a type-II isotherm, characteristic of macropores (diameter $>50 \mathrm{~nm}$ ). Hysteresis loops found were of type A. Such hysteresis is principally due to cylindrical pores (38).

Table III shows that the $S_{\mathrm{BET}}$ specific surface ared for A6 is approximately $60 \%$ higher than the geometrical specific surface area $S_{\mathrm{G}}$, while $S_{\mathrm{BET}}$ for the systems Mix 3 and Mix $3 \mathrm{~A} 2$ is almost equal to $S_{\mathrm{G}}$. $S_{\mathrm{G}}$ was calculated using a radius of $108 \mathrm{~nm}$ for A6, $63 \mathrm{~nm}$ for Mix 3 and a radius of $64 \mathrm{~nm}$ for Mix3A2 (see Table 2); the density used is given in Table 6 . A spherical shape was assumed.
The differences in the calculated and measured specific surface areas for A6 may arise from an underestimation of the surface area due to surface roughness of the spheres, or from the internal microporosity of the particles.

Although some surface roughness is clearly present on the particles, see Fig. 1, it was argued in the paper of Lecloux et al. (39) that the high value of $S_{\mathrm{BET}}$ compared to $S_{\mathrm{G}}$, as was measured and calculated by them for particles similar to A6, is due to the presence of micropores. They based this conclusion on the agreemenl they found between the cumulative specific surface area $S_{\text {cum }}$, without a correction for the micropores, and the calculated $S_{\mathrm{G}}$. This is indeed exactly what we find for A6 as well (see Table 3). However, for Mix 3 and Mix $3 \mathrm{~A} 2, S_{\text {cum }}$ does not equal $S_{\mathrm{G}}$, although $S_{\text {cum }}$ is smaller than $S_{\mathrm{BET}}$. Instead, for Mix 3 and Mix $3 \mathrm{~A} 2 S_{\mathrm{BET}}$ agrees very well with the value of $S_{\mathrm{G}}$. The fact that $S_{\mathrm{cum}}$, without a correction for the micropores, is smaller than $S_{\mathrm{BET}}$ for these two particles is in agreement with the larger micropore volume $V_{\mathrm{m}}$ of Mix3 and Mix $3 \mathrm{~A} 2$ compared to A6. It thus seems, that for Mix 3 and Mix $3 \mathrm{~A} 2$ an extra contribution from the micropores is included in $S_{\mathrm{BET}}$ as well, but for reasons that are not clear, the $S_{\mathrm{BET}}$ values are too low. It is a strange coincidence, however, that the too low $S_{\mathrm{BET}}$ values are so close to the calculated $S_{\mathrm{G}}$ values.

The agreement between the specific surface areas as obtained from the t-plots, $S_{\mathrm{t}}$, and $S_{\mathrm{RFT}}$ is reasonably good. The surface area of the micropores is included in $S_{1}$ as was also observed by Lecloux et al.

The $C_{\mathrm{BET}}$ constant, which describes the adsorption interaction of a nitrogen molecule with the surface, clearly reflects the differences in the surface properties of the silica systems. The value for the constants of $\mathrm{A} 6$ and Mix $3 \mathrm{~A} 2$ are signifcantly larger than that of Mix3. As was also demonstrated with the reaction of salicylcaldehyde with Mix 3 and Mix $3 \mathrm{~A} 2$, the surface of the Mix 3 spheres is partly covered with organic groups, while these groups are completely shielded from the solvent by the thin silica coating on Mix3A2. The aminopropyl groups reduce the adsorbent-adsorbate interactions. These groups are also known to decrease the surface tension of a coated silica surface (8).

The measured pore volumes $V_{\mathrm{p}}$ and $V_{\text {cum }}$ are close together, but too small for even a closed packed arrangement of the spheres. With a density of $2 \mathrm{~g} / \mathrm{cm}^{3}$ and without microporosity the free volume between $1 \mathrm{~g}$ of silica spheres would have been $0.13 \mathrm{~cm}^{3}$. The large radius of the particles is probably responsible for the missing pore volume. The pore distributions obtained showed maxima around 40-50 $\mathrm{nm}$. These mesopores, almost macropores, are so large that the hystcresis loop is confined in a region of relative pressures close to one. This explains why the voids between the particles with the largest pores are underestimated.

Micropores with radii of approximately $1.5 \mathrm{~nm}$ were also found. Because the particles investigated are relatively large, 


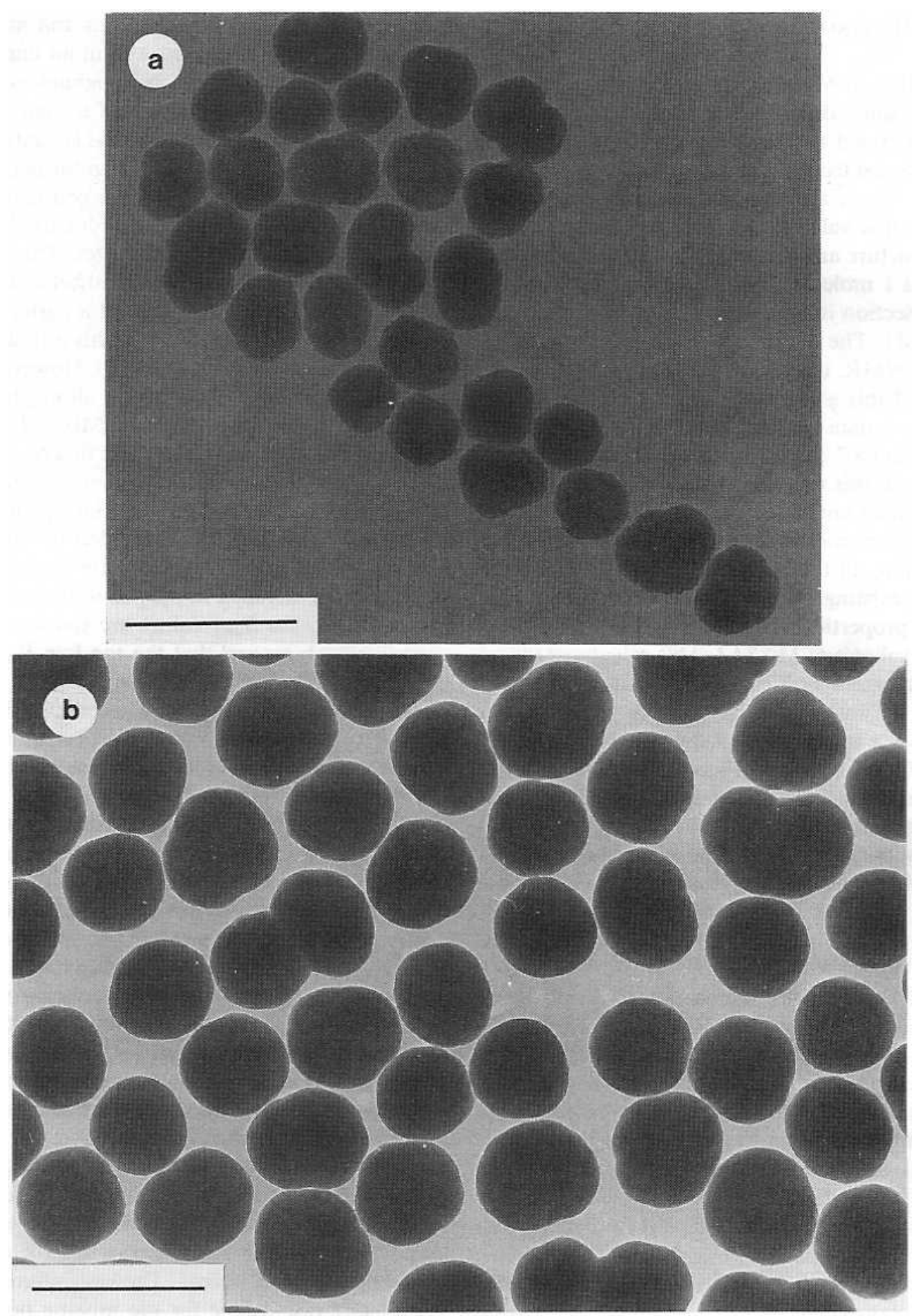

FIG. 1. Transmission electron micrographs. (a) Mix3A2S (bar $250 \mathrm{~nm}$ ). (b) Mix 3A3S (bar $250 \mathrm{~nm}$ ). (c) Mix4 (bar $1 \mu \mathrm{m}$ ). (d) Mix7 (bar $250 \mathrm{~nm}$ ).

the pore volume attributed to these internal micropores is small. In the paper of Lecloux, who described nitrogen adsorption measurements as a function of the radius of Stöber silica, the micropore volume was found to increase with de- creasing particle radius (39). The finding of micropores in the particles corroborates carlier assumptions of Van Helden et al. concerning the presence of pores smaller than $2 \mathrm{~nm}$. $\Lambda s$ could be expected from the density of the particles, the 

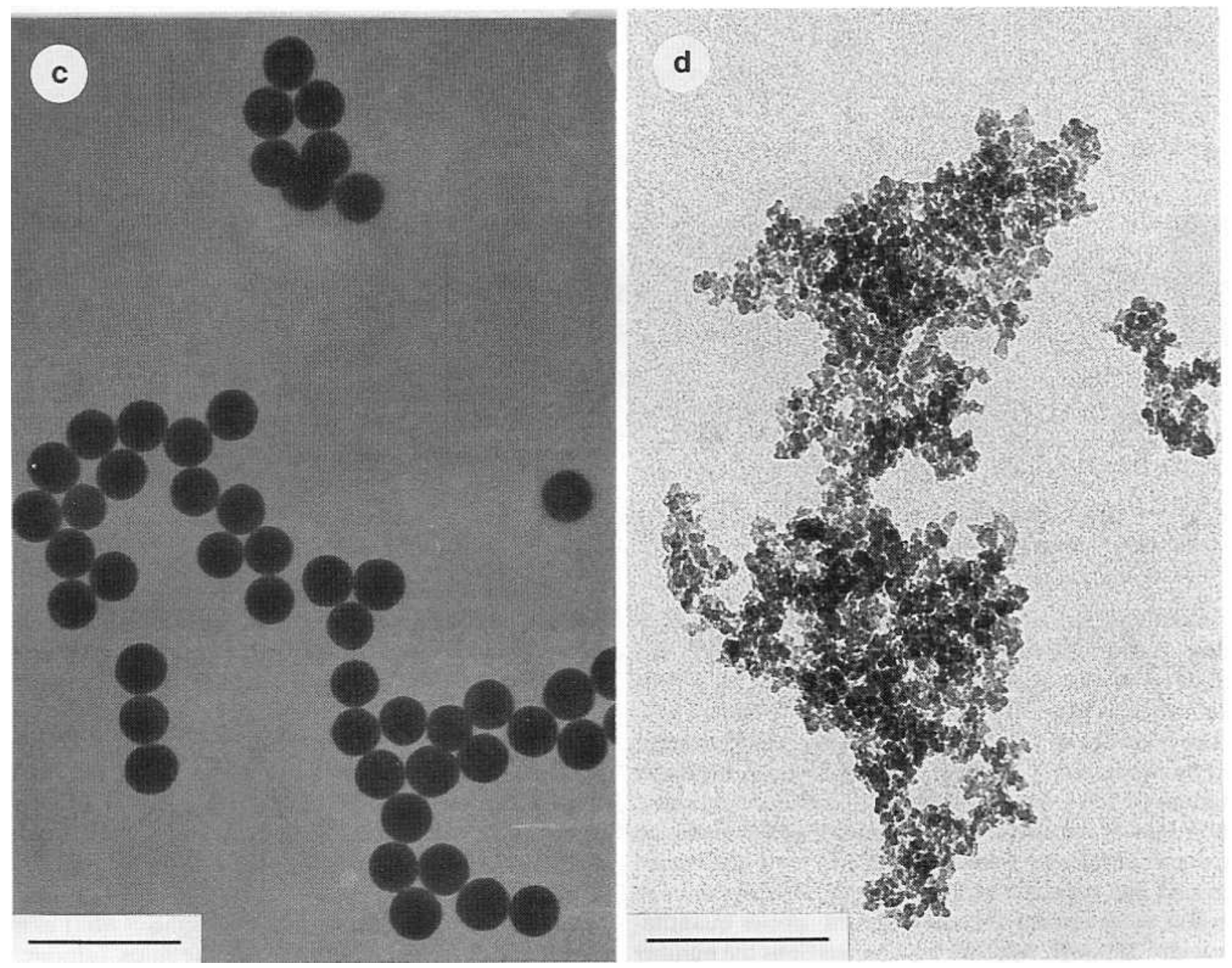

FIG. 1-Continued

micropore volume was larger for the organo-silica particles. The silica structure of the thin layer on Mix 3A2 still appeared more porous than the silica spheres of A6. These conclusions are, however, best repeated with smaller silica and organosilica spheres, where the larger specific surface area and micropore volume will allow more accurate determinations.

\section{Particle Composition}

${ }^{20}$ Si solid-state NMR. In Fig. 2a, a CP spectrum is shown in which almost all the different $\mathrm{Q}$ and $\mathrm{T}$ silicons can be distinguished. The organo-silica shown in Fig. $2 \mathrm{a}$ is not colloidal, but was made by allowing an equal volume mixture of TES and APS to hydrolyze and condense by atmospheric water during two hours to hybrid gel. In the CP spectrum of the APS-coated silica spheres A1APS, Fig. 2b, only the more condensed silicon atoms of the coupling agent and silica core can be recognized $\left(\mathrm{T}^{2}\right.$ at $60.5 \mathrm{ppm}, \mathrm{T}^{3}$ at 65.7 ppm, $\mathrm{Q}^{3}$ at $102.4 \mathrm{ppm}$, and $\mathrm{Q}^{4}$ at $\left.110.4 \mathrm{ppm}\right)$. These chemical shift values of the different silicon atoms are in agreement with literature values found for APS-modified silica (17, 18, 43 ) and were also found for the organo-silica spheres Mix 1.

The spin-lattice relaxation times for the silicon nuclei of the organo-silica spheres Mix 1 were determined by CP excitation. Since CP excitation is selective, the determined $T_{1 \mathrm{~S}}$ values are only an indication of the spin-lallice relaxation times that can be found in the different samples. As has been shown in the literature (31) there is even a distribution of relaxation times for silicon atoms with the same $\mathrm{Q}^{x}$ designation. These differences in rclaxation bchavior can be understood by realizing that on a molecular level these silica systems can be locally very different. If for instance, a $Q^{2}$ atom is close to a $\mathrm{T}^{x}$ silicon atom, the dipolar interactions due to the protons of the coupling agent may provide a more efficient relaxation than will be available to a $Q^{2}$ silicon atom surrounded by only $\mathrm{Q}^{4}$ silicons. 

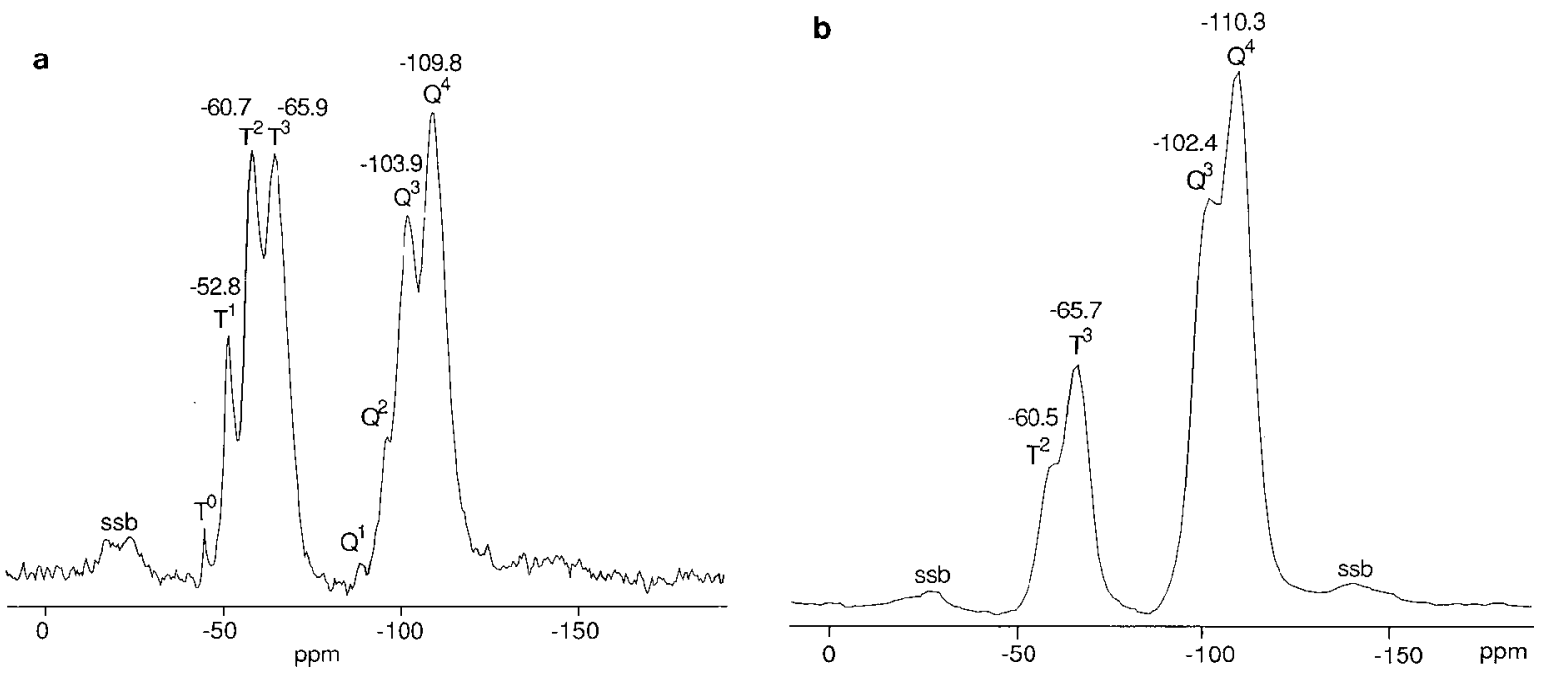

FIG. 2. ${ }^{29} \mathrm{Si} \mathrm{CP-MAS} \mathrm{NMR} \mathrm{spectra.} \mathrm{(a)} \mathrm{Equal} \mathrm{volume} \mathrm{mixture} \mathrm{of} \mathrm{TES-APS,} \mathrm{hydrolyzed/condensed} \mathrm{for} 2 \mathrm{~h}$ by atmospheric water ( 500 acquisitions). (b) AIAPS (13000 acquisitions).

As was found before (19) the logarithm of the integrated intensity versus time curves were convex, an indication of a distribution of relaxation times. An estimate of the largest relaxation times gave $70 \mathrm{~s}$ for $\mathrm{Q}^{3}, 90 \mathrm{~s}$ for $\mathrm{Q}^{4}$, and $50 \mathrm{~s}$ for $\mathrm{T}^{3}$. The intensity of $\mathrm{T}^{2}$ was too low to give reliable results. Based on the largest $T_{1 \mathrm{Si}}$ values, a delay between the direct excitations of $300 \mathrm{~s}$ was chosen for the quantitative spectra.

The result of the deconvoluted and integrated Gaussian peak intensities are presented in Table 8. Only the coated spheres A1 had a specific surface area that was large enough to be able to determine the chemisorbed species quantitatively with ${ }^{29} \mathrm{Si}$ NMR. After the coating reactions the number of $\mathrm{Q}^{2}$ and $\mathrm{Q}^{3}$ silicons of the silica core have decreased, although the difference is close to the estimated error. The

TABLE 8

Relative Intensities of the Different Types of Silicon Environments

\begin{tabular}{lccccc}
\hline SYSTEM & $\mathrm{Q}^{4}$ & $\mathrm{Q}^{3}$ & $\mathrm{Q}^{2}$ & $\mathrm{~T}^{3}$ & $\mathrm{~T}^{2}$ \\
\hline A1 $^{b}$ & $73^{a}$ & 26 & 1.7 & & \\
A1APS & 67 & 21 & - & 7.2 & 4.4 \\
& $(76)^{c}$ & $(24)$ & & $(62)$ & $(38)$ \\
A5 & 64 & 31 & 5.5 & & \\
A5APS & 65 & 30 & 4.0 & - & - \\
A6 & 66 & 30 & 4.2 & & \\
Mix1 & 40 & 31 & 3.8 & 19 & 7.2 \\
& $(53)$ & $(41)$ & $(5.1)$ & $(73)$ & $(27)$ \\
\hline
\end{tabular}

\footnotetext{
${ }^{a}$ Estimated errors for $\mathrm{Q}^{4}$ and $\mathrm{Q}^{3}: \pm 2$ for the other species $\pm \mathbf{1}$.

${ }^{b}$ The same reaction procedure as for the coating was followed, except for the addition of APS.

${ }^{c}$ Values are percentages related to Q-silicon and T-silicon atoms considered separately.
}

increase in the number of $Q^{4}$ groups is the result of condensation reactions with the coupling agent. The number $T$ silicon atoms on the surface is larger than the change in $\mathrm{Q}^{2}$ and $\mathrm{Q}^{3}$ species. Therefore, some of the APS molecules have also formed condensation products with other APS molecules. Nevertheless, if one assumes that all the TES has been used in the formation of the silica cores, which is not a bad assumption considering the low solubility of silica, only $4 \%$ of the APS used in the coating ended up on the particles (see Table 1).

In the synthesis of the organo-silica spheres Mix 1 as much as $37 \%$ of the APS ended up inside the silica, forming $26 \%$ of all the silicon nuclei in the particle. The relatively low content of $\mathrm{Q}^{4}$ and high content of $\mathrm{Q}^{3}$ and $\mathrm{Q}^{2}$ are a clear indication that the APS is not present as a thick surface layer, but is incorporated through (a part of) the particle interior. The aminopropyl groups inside the particle prevented the formation of a more condensed Q-siloxane structure. The T-siloxane structure is more condensed compared with the APS layer on A1, because the possibilities to react with Q species were higher for APS groups inside a particle.

${ }^{13} \mathrm{C}$ solid-state NMR. In the reaction products of APS and TES three different ethoxy groups are possible: those bonded to an APS or TES molecule and those belonging to a hydrolyzed or solvent ethanol molecule, which is physisorbed with hydrogen bonds to the condensation products. All three possibilities can be found in Fig. 3a, containing the CP-MAS spectrum of the above mentioned non colloidal condensation product of an APS and TES mixture. The assignments of the carbons bonded with oxygen of the three diffcrent cthoxy groups (Fig. 3a) was made with the help of the liquid-state spectra in which the hydrolysis of APS and TES in propanol was followed. 

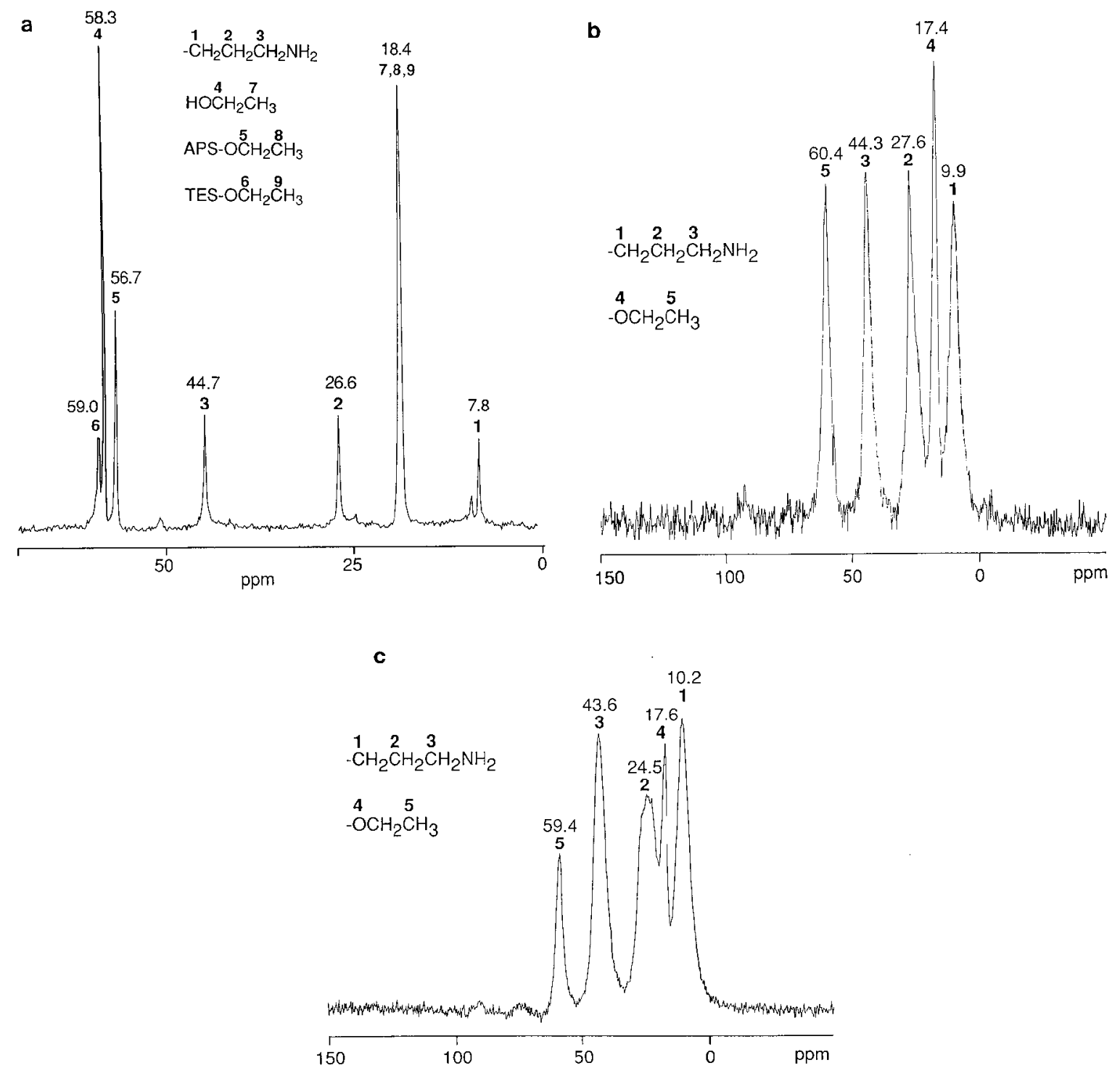

FIG. 3. ${ }^{13} \mathrm{C}$ CP-MAS NMR spectra. (a) Equal volume mixture of TES-APS, hydrolyzed/condensed for $2 \mathrm{~h}$ by atmospheric water (500 acquisitions). (b) AIAPS (3000 acquisitions). (c) Mix I (12000 acquisitions).

In all the other ${ }^{13} \mathrm{C} \mathrm{CP}$ spectra of the silica or organo-silica spheres only one type of ethoxy signal was observed, with peaks close to 60 and $18 \mathrm{ppm}$. These peaks are assigned as before (19) to ethoxy groups that never hydrolyzed from a TES molecule.

An interesting shift in the line position of the $\mathrm{C} 2$ carbon of the aminopropyl chain can be observed in the CP-MAS spectra of A1APS and Mix1 (Figs. 3b and 3c). In Fig. 3b, AlAPS, this carbon has a chemical shift of $27.6 \mathrm{ppm}$ and in Fig. 3c, Mix 1, the peak lies at $24.5 \mathrm{ppm}$ and is clearly broader. Sudhölter et al. (17), Chiang et al. (13a) and, quite extensively, Caravajal et al. (43) have investigated this shift in the resonance position of the $\mathrm{C} 2$ carbon of APS on silica gel.
Chiang et al. used electric field theory to calculate the effect of hydrogen bonding and the formation of an internal zwitterion on the chemical shift of this carbon atom. Sudhölter et al. and Caravajal et al. showed by reacting the APS with $\mathrm{HCl}$ that the hydrogen-bonded APS had a $\mathrm{C} 2$ chemical shift close to $22 \mathrm{ppm}$ and free APS had a resonance in the range 26-29 ppm. These observations werc in agrecment with the calculations and experimental observations of Chiang et al. and are also the explanation for the observed shift in Fig. 3. Aminopropyl chains inside the Mix 1 spheres are partly hydrogen bonded to silanol groups that are available because the siloxane structure is far from being fully condensed. The hydrogen bonding causes the decrease in chemical shift of 
the $\mathrm{C} 2$ carbon and broadens the peak, because not all the amine groups are hydrogen bonded and/or because the bonded molecules are more restricted in their movements (see Ref. (43)). On the surface of the more condensed Al particles there are less free silanol groups available for hydrogen bonding and most of the amine groups are free. Thus, the chemical shift of the $\mathrm{C} 2$ carbon of the propyl chain of APS provides useful information on the status of the amino group.

Although the signals were weak compared to the ethoxy signals at 60 and $18 \mathrm{ppm}$, the aminopropyl peaks could be detected with $\mathrm{CP}$ on the coated spheres of A5 and A6. As expected the chemical shifts for the carbons of the propyl chain were close to that of A1APS.

Elemental analysis. No elemental analysis for silicon and oxygen was done, therefore it is necessary to use the quantitative ${ }^{29} \mathrm{Si}$ NMR results to be able to check the elemental analysis results for internal consistency. By subtracting the weight percentages of hydrogen, carbon and nitrogen, one obtains the fraction of one gram of (organo)-silica that consists of the elements silicon and oxygen. For pure silica $\left(\mathrm{SiO}_{2}\right)$ this quantity can be converted to $n$ moles, using the molecular weight. However, the silica's studied do not consist of pure $n \mathrm{SiO}_{2}$, but consist of $n \mathrm{SiO}_{x}$. The $x$ can be calculated using the relative $\mathrm{Q}$ and $\mathrm{T}$ intensities from Table 8. For instance, if the silica would consist of only $\mathrm{Q}^{2}$ species the $x$ would be 3 , because in this structure there are three oxygen atoms for every silicon atom. In this way the number of moles TES and APS that were incorporated in one gram of (dried) silica can be calculated.

The analysis of the number of ethoxy groups in the silica spheres has been discussed before (19). Here we want to compare the number of APS groups calculated from the Tsilicons with the numbers calculated from the amount of nitrogen present in the samples.

For $1 \mathrm{~g}$ of AIAPS the number of APS molecules calculated from the number of silicon atoms is $1.68 \mathrm{mmol} ; 1.56 \mathrm{mmol}$ is calculated from the nitrogen percentage. The difference between these values may be explained by assuming that a few percent of $\mathrm{H}_{2} \mathrm{O}$ was also present in the particles. The agreement between the amount of APS calculated for the other silica system containing a relative large amount of APS, Mix 1, is even better. One gram of organo-silica Mix 1 contains $3.28 \mathrm{mmol}$ APS as calculated from the siloxane structure and $3.19 \mathrm{mmol}$ as calculated from the weight fraction of nitrogen. Again some water may be present as well, explaining the somewhat lower value obtained from the number of nitrogen atoms.

Another check for consistency of the data can be performed by using the $n \mathrm{SiO}_{x}$ structure and carbon and nitrogen data to calculate the percentage of hydrogen per gram. In this calculation it is assumed, that all the ethoxy groups are bonded with a silicon atom and are not present as ethanol (see the ${ }^{13} \mathrm{C}$ NMR results). In all cases a reasonable agreement is found between the measured and calculated hydrogen percentages. Mostly an amount of 0.1 to $0.2 \%$ is not accounted for by the calculated values. Again this points to the presence of a small amount of physically adsorbed water.

With the particles radius as given in Table 2 and the density from Table 5 a specific surface area can be calculated, which can be used together with the nitrogen percentage to obtain the number of APS groups per $\mathrm{nm}^{2}$. The number of silanol groups per $\mathrm{nm}^{2}$ is usually taken to be 4.5 for silica (23).

By taking the radius of $\mathrm{A} 5$ to be $72 \mathrm{~nm}$ the surface coverage of A5APS becomes 6.5 groups per $\mathrm{nm}^{2}$. For the coverage of A1APS a value of 5.6 per $\mathrm{nm}^{2}$ is calculated. The small difference in these values suggest, that the silica surface available to the coupling agent determines the amount that is taken up per particle. By also using the data from the quantitative titrations (Table 4) a value of 6.8 groups per $\mathrm{nm}^{2}$ can be calculated for A6APS.

These surface coverages can be compared with data reported by Philipse et al., who covered the same kind of silica spheres with the coupling agent methacryloxypropyltrimethoxysilane (5). A surface coverage of 10 groups per $\mathrm{nm}^{2}$ was reported, which is, considering the fact that a different coupling agent was used, close to the values found by us for APS.

Badley et al. have also modified "Stöber" silica with several coupling agents, among which was APS, using a procedure that was similar to that described by us for the synthesis of A6APS (6). By using their TEM radius, a surface coverage for APS of 15 groups per $\mathrm{nm}^{2}$ is calculated. This coverage is twice as high as compared to the values obtained by us, although there were no important differences in the coating conditions. Perhaps the difference is caused by the different procedure they used to separate the particles from the solvent. We used centrifugation and drying, they precipitated the spheres by acidifying the dispersion to $\mathrm{pH} 2$ with $\mathrm{HCl}$. It is possible that this acid treatment causes and additional deposition of APS on the silica. It is known that condensation reactions for organoalkoxysilanes are accelerated in acid-catalysis relative to the rate in base-catalysis (see the Theoretical section).

Determination of APS. The absorption spectrum of the salicylaldimine that was made from APS and SA in ethanol contained a maximum at $405 \mathrm{~nm}$, the molar extinction coefficient determined at this maximum was $995 \mathrm{dm}^{3} /(\mathrm{mol} \mathrm{cm})$. However, the extinction coefficient appeared to depend on the degree of hydrolysis of the APS. In mixtures of water and ethanol the extinction coefficient decreased $10 \%$ during a day and remained approximately constant thereafter (see also the hydrolysis experiments in the section on NMR). Because APS bound on the surface of silica consists of a whole range of molecules with different siloxane bonds and number of silanol groups, and thus different extinction coef- 
ficients, the salicylaldimine could not be used in a quantitative determination.

The qualitative reaction of SA with APS on the silica spheres proved to be a very simple test to the presence of amine groups on the surface. If the silica particle was colored yellow in solution, the amine groups could always also be detected on dried silica.

It is no surprise that APS was found on A2APS, A5APS and A6APS. All the Mix particles turned even more yellow upon treatment with SA. More interesting is the fact that the amine groups of Mix $3 \mathrm{~S}$ could still be detected even in cyclohexane. The test for the Mix $3 \mathrm{~A} 1$ particles was also positive, which indicates that (part of) the amine groups could still be reached. For Mix3A2 and the other particles with core Mix 3 the test was negative, because now the APS groups were buried inside the particles.

The quantitative results obtained by the titration of adsorbed $\mathrm{Cl}^{-}$are given in Table 4 . The values seem to be slightly smaller than the amounts obtained with elemental analysis, but the agreement is good considering the differences in the techniques. Perhaps a slight hydrolysis of the APS groups from the particle surface during the treatments with water is responsible for the small differences observed.

In the coating of large particles with a relativcly small specific surface area, the sensitivity of the titration method for APS is much larger than NMR and also still importantly larger than elemental analysis. A disadvantage is, that the method is laborious.

\section{Particle Properties}

Particle density and refractive index. Densities and refractive indices arc given in Tablc 5 . The difference in particle density of the organo-silica spheres Mix 1 and Mix 3 compared to the silica particles A5 and A6 is quitc large. Although the amount of APS taken up in the Mix2 particles was not determined, the increased density compared to Mix 1 and the lower amount of APS used in the synthesis, indicate that the content of APS is probably lower than in Mix 1 and Mix 3 .

The increase in density going from Mix 3 to Mix $3 \mathrm{~A} 2$ seems somewhat large considering the relatively small amount of TES added in the coating reaction. The density of Mix $3 \mathrm{~A} 3$ is, however, in good agreement with the measured increase in radius: By taking a radius of $63 \mathrm{~nm}$ for Mix 3 and $79 \mathrm{~nm}$ for Mix $3 A 3$ and using a density for the silica layer of $2 \mathrm{~g} /$ $\mathrm{cm}^{3}$, the calculated value for the density of Mix $3 \mathrm{~A} 3$ is $1.78 \mathrm{~g} / \mathrm{cm}^{3}$.

The data for the refractive indices of the different systems are unexpected, because they all indicate that the organosilica particles have a slightly higher refractive index than the particles prepared from TES alone. It was expected that the refractive index of organo-silica would be lower than that of "Stöber" silica, because the density of organo-silica is lower. For silica prepared with the Stöber method a lower density is accompanied by a lower refractive index compared to the values reported for amorphous silica (23).

The particle refractive index was measured in mixtures of solvents (Table 5) to be able to change the solvent refractive index over a large range. However, in a mixture of solvents there is always the possibility of preferent adsorption of one of the components onto the particle surface (see (2a)). I herefore, different mixtures of solvents were used to observe effects of preferent adsorption.

Preferent adsorption is probably the reason for the systematically lower values found in the mixture of 2-bromoethanol-ethanol compared to ethanol-toluene. In cyclohexane/toluene the values are again different, but here the octadecanol coating also influences the results. It should also be mentioned that the colloidal systems were not stable in the ethanol-toluene mixtures, in which they formed a gel phase, and that the Mix3A2S was not stable in the cyclohexane-toluene mixture either. These instabilities did not influence the determination of the particle refractive index, because a clear minimum in the scattering was still observed and at this minimum the turbidity almost disappeared just as it did with the stable dispersions in 2-bromoethanolethanol.

Still, in all the solvents mixtures and also with the octadecanol coated particles the refractive index of the organosilica spheres was found to be higher. Furthermore, the organo-silica particle refractive index decreased when the particles were coated with layers of silica with increasing thickness (Mix3A2S and Mix3A3S, Table 5). Although the increase in particle refractive index is not large, these results seem to indicate that the mean refractive index of the particles increases upon the incorporation of APS.

Particle stability. Particle mobilities and the limiting concentrations of $\mathrm{LiNO}_{3}$ that resulted in a stable dispersion in $1.1 \mathrm{M} \mathrm{NH}_{3}$ and $3.0 \mathrm{M} \mathrm{H}_{2} \mathrm{O}$ are given in Table 7. The stability of an $\Lambda P S$ coated particle, A6APS, and an organosilica sphere of the about the same size, Mix 1, were compared with an uncoated silica sphere, A6, in order to investigate the influence of the presence of APS on the particle surface. The particle mobilities were not converted to $\xi$-potentials, because several physical parameters of the solvent mixture are not known; these properties are the viscosity, the dielectric constant and most importantly the dissociation constant of ammonia. Still, the mobilities can be used to compare the particle charge or surface potential, because the solvent composition was the same for the systems collected in Table 7 and the particle radii are close together. The decreased mobilities of the spheres with APS on the particle surface indicate, that the negative charge on the colloids is reduced. The acidity of a silanol group bonded to a T-silicon atom is lower than the acidity of a silanol belonging to a $Q$ silicon. As explained in the theoretical section, this decrease in the dissociation of the silanol groups is caused by the decrease 
in positive charge on silicon if a siloxane bond is replaced by a carbon-silicon bond. Another factor that is probably decreasing the net particle charge, is the hydrogen bonding capacity of the amine group. As was also indicated by the ${ }^{13} \mathrm{C}$ measurements and by the increased stability of APS molecules in water, the formation of a hydrogen bond, or even the transfer of a proton, between a silanol group and amine group can also decrease the net negative charge $\left(-\mathrm{SiO} \cdot \mathrm{H}_{3} \mathrm{~N}-\right)$. The decreased surface potential of the particles with amine groups on the surface is also reflected in the low concentrations of $\mathrm{LiNO}_{3}$ that could be added without losing the colloidal stability (Table 7).

Another direct consequence of the reduced charge on spheres with APS on their surface and the result of the increased sensitivity to the ionic strength, was the flocculation of the Mix 3 particles when too much TES was added too quickly. Bogush and Zukoski (44) have shown that in the first few minutes after the addition of TES the conductivity of the reaction mixture sharply increases and that during the rest of the reaction (taking hours) it slowly decreases back to its initial value. The increase of the conductivity is caused hy the dissociation of hydrolyzed silanol groups and this increase in ionic strength causes the flocculation of the APS coated particles. By keeping the concentration of TES low until all the amine groups are buried inside the particle, the flocculation can be prevented and the subsequent growth can proceed with high TES concentrations just as with "ordinary" silica particles (9).

The stability of the different, octadecanol-coated spheres in three solvents is given in Table 4 . The stability criterion was defined in the experimental section. In the following sections some (tentative) explanations for the observed differences will be presented.

The instability of Mix $3 \mathrm{~S}$ in cyclohexane and toluene could be due to a reduced density of the stabilizing octadecyl chains on the particle surface. The number of octadecyl chains is probably lower than on regular silica, because the amine groups on the surface could still be reached by the SA molecules after the coating reaction. Thus, the formation of hydrogen bonds between the amine groups of different particles is probably also possible.

This reasoning, however, does not hold for the system Mix2A2S, which was also unstable in cyclohexane and toluene. Here no yellow color appeared after treatment with SA, which indicated that the amine groups could not be reached anymore by the aldehyde. Since the same particle with a somewhat thicker layer of silica, Mix3A3S, was stable in cyclohexane, a different explanation is necessary to explain the attractions between the particles of Mix3A2S. More work has to be done to understand this in detail, but it is certainly interesting to notice that such subtle differences on the particle surface influence the particle stability.

The remarkable stability of all the coated Mix 3 particles in chloroform is contributed to charge on the particles in this slightly more polar solvent (see Ref. (12) for strong indications of a charge on stearylsilica in chloroform).

\section{E. Mechanism of Surface Coating and Growth of Organo-silica Spheres}

In the experimental section on NMR two experiments are described in which the hydrolysis of TES and APS was followed. The first order hydrolysis constants $\left(k_{\mathrm{h}}\right)$ were obtained in propanol (concentrations given in the experimental section ). The values obtained were $k_{\mathrm{h}}=6.0 \times 10^{-3} \mathrm{~min}^{-1}$ for TES and $k_{\mathrm{h}}=1.2 \times 10^{-3} \mathrm{~min}^{-1}$ for APS.

These quantitative results illustrate the effect of the substitution of a $\mathrm{Si}-\mathrm{O}$ bond with a $\mathrm{Si}-\mathrm{C}$ bond already mentioned in the theoretical section. Because of the electron donating capacity of the alkyl group, the positive charge on the silicon atom is reduced and the attack of the catalytic hydroxide ion is less likely. Besides this inductive effect, which is present in all organo-trialkoxysilanes, there is an additional steric hindering caused by the proposed internal ring formation of the 3-aminopropyl derivative.

There is no reason to expect that this large difference in hydrolysis rate between TES and APS will change going from propanol to ethanol. The slow hydrolysis of APS could have had the following implications for the base-catalyzed formation of organo-silica: When all the TES is hydrolyzed and converted into silica the amount of hydrolyzed APS would still be small. Since the reaction rate between a hydrolyzed TES molecule and a hydrolyzed APS molecule is likely (much) smaller than that between two hydrolyzed TES molecules, the amount of APS incorporated into the silica would be very small. These expected consequences from the differences in hydrolysis rate between tri- and tetraalkoxysilanes was the reason why it was argued in the literature, that hybrid silica materials could best be produced through acid-catalyzed reactions (22). With acid catalysis, however, no stable colloids are formed.

Thus, the production of organo-silica spheres where $26 \%$ of the silicon atoms belong to the coupling agent is surprising. Together with the results of the coating reactions, this unexpected result contains strong clues to the mechanism of the incorporation of APS.

The quantitative results obtained in the coating of A1 and A5 (Table 4) demonstrate, that once the surface of silica has been coated with APS, the further addition of APS stops or goes very slowly. This result was also found for A6APS where all the APS, or at least an important part of it, was hydrolyzed during the long reaction time. The resistance of a hydrolyzed APS molecule to condensate with another hydrolyzed silanol group belonging to a $\mathrm{T}$-silicon atom was also observed in the formation of Mix 7, where after one day no structures were formed that scattered light. 
The incorporation of APS in the Mix systems is therefore proposed to proceed for an important part through an alcohol producing condensation of APS with a silanol group belonging to a Q-silicon atom. The alcohol producing condensation of APS can take place directly on the surface (with $Q^{2}$ or $Q^{3}$ ) of a particle, which also grows larger through condensation with hydrolyzed TES monomers, or the condensation reaction can take place in solution with a hydrolyzed TES monomer (or small oligomer), which subsequently attaches itself to the surface of a growing sphere. The importance of either path depends not only on the concentration, which is probably higher for the hydrolyzed monomers, but also on the reaction rate, which is higher for the more acidic surface silanol groups.

In the synthesis of Mix 7 most of the APS was already hydrolyzed when TES was added. Therefore, water producing condensation between a hydrolyzed TES molecule and APS could also take place. The result was, that the amount of APS that reacted with hydrolyzed TES was too high to obtain stable colloidal particles (Fig. 1d). The reason could be that the resulted structures were polymer like, even less condensed, than the Mix 1 particles and/or that the charge on the resulting structures was too low to prevent coagulation. The results obtained in the synthesis of Mix 7 further demonstrate the necessity of removing the partly hydrolyzed APS after formation of the organo-silica spheres, if these particles are to be covered by a layer of silica.

That the incorporation of APS takes place through an alcohol producing condensation has also consequences for the mechanism of particles formed from TES alone. So far, alcohol producing condensation reactions have not been considered to be of any importance in the proposed models for the formation and growth of silica spheres. The results obtained with APS suggest otherwise. If alcohol-producing condensations are important in the reactions between TES molecules as well, it would help to explain why ethoxy groups that belong to incompletely hydrolyzed TES molecules are found inside the silica spheres (19).

Because APS is extra hindered in its hydrolysis and condensation reactions, the fact that it is incorporated rather easily strongly suggests that the same will be true for other coupling agents as well.

\section{CONCLUSIONS}

In this paper it has been shown, that silica spheres prepared from tetraethoxysilane (TES) in mixtures of water, ammonia and ethanol can be coated by the silane coupling agent 3aminopropyltriethoxysilane (APS) by a simple procedure in the same reaction mixture in which the silica particles were made. It has also been shown, that if the synthesis of colloidal particles is started from mixtures of TES and APS spherical, monodisperse particles can be made that contain a suhstan- tial amount of organic groups. The amount of groups incorporated into the particles depends on the amount of APS used, but more work is needed to be able to make a quantitative prediction on the composition or final particle radius of the composite particles.

These new kind of organo-silica spheres differ in several aspects from silica spheres made from TES alone: the negative surface charge is smaller, the density is lower (for instance $1.51 \mathrm{~g} / \mathrm{cm}^{3}$ as opposed to $2.03 \mathrm{~g} / \mathrm{cm}^{3}$ ), the siloxane structure is less condensed, and the microporosity is higher. The refractive indices of the organo-silica spheres are somewhat higher $(\approx 0.02)$, but the differences are small.

It has also been shown, that a layer of silica of any desired thickness can be grown onto the organo-silica spheres. The possibility to deposit silica onto the hybrid particles makes this kind of colloidal spheres even more suited as a colloidal model system, because now organo-silica spheres coated with a layer of silica can be used as a tracer in a dispersion of "normal" silica particles. Such a mixture of particles would form a real tracer system. Now the interparticle interactions are almost completely determined by the surface coating and the outer silica layer. For instance, the different elemental composition of the organo-silica spheres could be exploited by a tracer study with small angle neutron scattering. Or the difference in the density of these spheres could be used in sedimentation experiments.

Also, the amine groups can be coupled to other groups that can subsequently be incorporated in the particle interior. The feasibility of this last example of the use of APS-organosilica spheres has been shown by the synthesis of fluorescent silica spheres $(11,12)$.

The proposed mechanism for the incorporation of APS is an alcohol producing condensation with a silanol group belonging to a Q-silicon atom. This Q-silicon atom can be part of a monomer or part of an already condensed structure. From this reaction pathway it is concluded that an alcoholproducing condensation may also be important in the production of silica spheres from TES alone. Certainly, these hypothesis have to be justified by further research.

We expect the base-catalyzed method to prepare organosilica as described in this paper to be applicable to mixtures of other tetraalkoxy and organo-trialkoxysilanes as well. We hope, therefore, that the results presented to make hybrid materials will not only be of interest to the colloids science, but will also be of importance to the materials science.

\section{ACKNOWLEDGMENTS}

G. Nachtegaal is thanked for her help with the NMR measurements at the SON-NWO HF-NMR facility (University of Nijmegen, the Netherlands). We appreciated the critical reading of the manuscript by A. P. M. Kentgens, working at the same facility. We also thank J. Suurmond and J. Pieters for making the electron micrographs, P. A. Elberse (Department of Inorganic 
Chemistry, University of Utrecht) for performing the nitrogen adsorption measurements and $\mathrm{M}$. Verweij for performing some of the experiments.

This work was supported by the Netherlands Foundation for Chemical Research (SON) with financial aid from the Netherlands Organization for Scientific Research (NWO).

\section{REFERENCES}

1. Stöber, W., Fink, A., and Bohn, E., J. Colloid Interface Sci. 26, 62 (1968).

2. (a) Van Helden, A. K., Jansen, J. W., and Vrij, A., J. Colloid Interface Sci. 81, 354 (1981); (b) Van Helden, A. K., and Vrij, A., J. Colloid Interface Sci. 76, 418 (1980).

3. Duits, M. H. G., May, R. P., Vrij, A., and de Kruif, C. G., Langmuir 7,62 (1991)

4. de Kruif, C. G., Jansen, J. W., and Vrij, A., in "Physics of Complex and Supcrmolecular Fluids" (S. A. Safran, and N. A. Clark, Eds.). Wiley, New York, 1987.

5. Philipse, A. P., and Vrij, A., J. Colloid Interface Sci. 128, 121 (1989).

6. Badley, R. D., Ford, W. T., McEnroe, F. J., and Assink, R. A., Langmuir 6, 792 (1990)

7. Pathmamanoharan, C., Colloids Surf. 50, 1 (1990)

8. Goodwin, J. W., Harbron, R. S., and Reynolds, P. A., Colloid Polym. Sci. 268, 766 (1990).

9. (a) Coenen, S., and de Kruif, C. G., J. Colloid Interface Sci. 124, 104 (1988); (b) Philipse, A. P., and Vrij, A., J. Chem. Phys. 87, 5634 (1987).

10. Schunid,, H., Scholee, H., and Kaiser, A., J. Non-Cryst. Solids 63, 1 (1984).

11. Van Blaaderen, A., Imhof, A., Hage, W., and Vrij, A., Langmuir 8, 1514 (1992)

12. Van Blaaderen, A., Peetermans, J., Maret, G., and Dhont, J. K. G., J. Chem. Phys. 96, 4591 (1992).

13. (a) Chiang, C. H., Liu, N. I., and Koenig, J. L., J. Colloid Interface Sci. 86, 26 (1982); (b) Culler, S. R.. Ishida, H., and Koenig. J. L., J. Colloid Interface Sci. 106, 334 (1985); (c) Naviroj, S., Culler, S. R., Koenig, J. L., and Ishida, H., J. Colloid Interface Sci. 97, 308 (1984).

14. Waddell, T. G., Leyden, D. E., and DeBello, M. T., J. Am. Chem. SoC. 103, 5303 (1981).

15. Murthy, R. S. S., and I eyden, D. E., Anal. Chem. 58, 1228 (1986)

16. Gimpel, M., and Unger, K., Chromatographia 16, 117 (1982).

17. Sudhölter, E. J. R., Huis, R., Hays, G. R., and Alma, N. C. M., J. Colloid Interface Sici. 103, 554 (1985).

18. De Haan, J. W., van den Bogaert, H. M., Ponjee, J. J., and van de Ven, L. J. M., J. Colloid Interface Sci 110, 591 (1986).

19. (a) Van Blaaderen, A., Van Geest, J., and Vrij, A., J. Colloid Interface Sci., in press; (b) Van Blaaderen, A., and Kentgens, A. P. M., J. NonCryst. Solids 149, 161 (1992).
20. Noll, W., "Chemie und Technologie der Silicone," 2nd ed., (pp. 1-15, 168-210, 246-287. Verlag Chemie GMBH, Weinheim, 1968.

21. Brinker, C. J., and Scherer, G. W., "Sol-Gel Science," 1st ed., pp. 108216. Academic Press, Boston, 1990.

22. Schmidt, H., Scholze, H., and Kaiser, A., J. Non-Cryst. Solids 63, 1 (1984).

23. Iler, K. K., "The Chemistry of Silica." Wiley, New York, 1979.

24. Lippert, J. L., Melpolder, S. B., and Kelts, L. M., J. Non-Cryst. Solids 104, 139 (1988).

25. Plueddemann, E. P., "Silane Coupling Agents." Plenum, New York, 1982.

26. Fyfe, C., "Solid State NMR for Chemists." C.F.C. Press, Ontario, 1983.

27. (a) Lippmaa, E. T., Alla, M. A., and Pehk, T. J., J. Am. Chem. Soc 100, 1929 (1978); (b) Lippmaa, E., Maegi, M., Samoson, A., Engelhardt, G., and Grimmer, A. R., J. Am. Chem. Soc. 102, 4889 (1980).

28. Glaser, R. H., Wilkes, G. L., and Bronnimann, E., J. Non-Cryst. Solids 113, 73 ( 1989).

29. (a) Maciel, G. E., and Sindorf, D. W., J. Am. Chem. Soc. 102, 7606 (1980); (b) Sindorf, D. W., and Maciel, G. E., J. Phys. Chem. 86, 5208 (1982); (c) Sindorf, D. W., and Maciel, G. E., J. Am. Chem. Soc. 105, $3767(1983)$

30. Fyfe, C. A., Gobbi, G. C., and Kennedy, G. J., J. Phys. Chem. 89, 277 (1985)

31. Pfleiderer, B., Albert, K., Bayer, E., van de Ven, L., de Haan, J., and Cramers, C., J. Phys. Chem. 94, 4189 (1990).

32. Weast, R. C. (Ed.), 56th ed. "Handbook of Chemistry and Physics," Chemical Rubber Co., Cleveland, 1976.

33. Philipse, A. P., Smits, C., and Vrij, A., J. Colloid Interface Sci. 129 335 (1989)

34. Koppel, D. E., J. Chem. Phys. 57, 4814 (1972).

35. Van Veluwen, A., Lekkerkerker, H. N. W., de Kruif, C. G., and Vrij, A., J. Chem. Phys. 89, 2810 (1988).

36. Bohren, C. F., and Huffman, D. R., "Absorption and Scattering of Light by Small Particles." Wiley, New York, 1983.

37. Pecora, R., (Ed.), "Dynamic Light Scattering: Applications of Photon Correlation Spectroscopy." Plenum, New York, 1985.

38. Lowell, S., and Shields, J. E., "Powder Surface Area and Porosity," second ed. Chapman and Hall, London, 1987.

39. Lecloux, A. J., Bronckart, J., Noville, F., Dodet, C., Marchot, P., and Pirard, J. P., Colloids Surf. 19, 359 (1986).

40. Torchia, D. A., J. Magn. Reson. 30, 613 (1978).

41. Hunter, R. J., "Zeta Potential in Colloid Science." Academic Press, London, 1981.

42. Brunauer, S., Deming, L. S., Deming, W. S., and Teller, E., J. Am Chem. Soc. 62, $1723(1940)$

43. Caravajal, G. S., I.eyden, D. E., Quinting, G. R., and Maciel, G. E., Anal. Chem. 60, 1776 (1988).

44. Bogush, G. H., and Zukoski, C. F., IV. J. Colloid Interface Sci. 142, 1 (1991). 